\title{
Computational Study of Metal Contacts to Monolayer Transition-Metal Dichalcogenide Semiconductors
}

\author{
Jiahao Kang, ${ }^{1}$ Wei Liu, ${ }^{1}$ Deblina Sarkar, ${ }^{1}$ Debdeep Jena, ${ }^{2}$ and Kaustav Banerjee ${ }^{1}$ \\ ${ }^{1}$ University of California, Santa Barbara, California 93106, USA \\ ${ }^{2}$ University of Notre Dame, Notre Dame, Indiana 46556, USA
}

(Received 27 June 2013; revised manuscript received 23 February 2014; published 14 July 2014)

\begin{abstract}
Among various 2D materials, monolayer transition-metal dichalcogenide (mTMD) semiconductors with intrinsic band gaps (1-2 eV) are considered promising candidates for channel materials in next-generation transistors. Low-resistance metal contacts to mTMDs are crucial because currently they limit mTMD device performances. Hence, a comprehensive understanding of the atomistic nature of metal contacts to these $2 \mathrm{D}$ crystals is a fundamental challenge, which is not adequately addressed at present. In this paper, we report a systematic study of metal-mTMD contacts with different geometries (top contacts and edge contacts) by ab initio density-functional theory calculations, integrated with Mulliken population analysis and a semiempirical van der Waals dispersion potential model (which is critical for 2D materials and not well treated before). Particularly, In, Ti, Au, and Pd, contacts to monolayer $\mathrm{MoS}_{2}$ and $\mathrm{WSe}_{2}$ as well as $\mathrm{Mo}_{-} \mathrm{MoS}_{2}$ and $\mathrm{W}-\mathrm{WSe}_{2}$ contacts are evaluated and categorized, based on their tunnel barriers, Schottky barriers, and orbital overlaps. Moreover, going beyond Schottky theory, new physics in such contact interfaces is revealed, such as the metallization of mTMDs and abnormal Fermi level pinning. Among the top contacts to $\mathrm{MoS}_{2}$, Ti and Mo show great potential to form favorable top contacts, which are both $n$-type contacts, while for top contacts to $\mathrm{WSe}_{2}, \mathrm{~W}$ or Pd exhibits the most advantages as an $n$ - or $p$-type contact, respectively. Moreover, we find that edge contacts can be highly advantageous compared to top contacts in terms of electron injection efficiency. Our formalism and the results provide guidelines that would be invaluable for designing novel 2D semiconductor devices.
\end{abstract}

DOI: 10.1103/PhysRevX.4.031005

\section{INTRODUCTION}

As silicon complementary metal oxide semiconductor technology approaches its limits in scaling, an alternative material to silicon is needed for future logic transistor applications. 2D crystal semiconductors, with extremely small thicknesses (few $\AA$ ), uniform band gap over a large area, and pristine interfaces without out-of-plane dangling bonds, have the potential to allow efficient electrostatics, reduction of short channel effects, fewer traps on a semiconductor-dielectric interface, and a high degree of vertical scaling (Fig. 1). Recently, many types of 2D materials, including graphene [1,2] and hexagonal boron nitride $(h$-BN) [3], have been experimentally and theoretically demonstrated. While these materials do not have the requisite band gaps $\left(E_{g}\right)$ [graphene: $0 \mathrm{eV} ; h$-BN: $>5 \mathrm{eV}$; see Fig. 2(a)] for use as channel materials in digital applications, monolayer transition-metal dichalcogenide (mTMD) semiconductors, a family of 2D semiconductor monolayers

Published by the American Physical Society under the terms of the Creative Commons Attribution 3.0 License. Further distribution of this work must maintain attribution to the author(s) and the published article's title, journal citation, and DOI.
Subject Areas: Computational Physics, Electronics, Nanophysics arranged in a hexagonal lattice [Figs. 2(b) and 2(c)], exhibit considerable $E_{g}(1-2 \mathrm{eV})$ [Figs. 2(a), 2(d), and 2(e)], thereby presenting great potential for low-power digital applications [4-7]. The 2D TMD materials are also attractive for display electronics [8] due to their inherent flexibility, transparency, and dangling-bond-free interface that make them easy to integrate with various substrates. Moreover, field-effect-transistor (FET-)based biosensors with 2D TMD semiconductor as the channel material have recently been demonstrated [9] to be highly advantageous over all other nanomaterial-based (including graphene) FET biosensors, due to their atomically layered and planar nature, nonzero band gaps, and pristine surfaces.

However, the formation of low-resistance metal contacts is the biggest challenge that masks the innate exceptional electronic and magnetic properties of 2D semiconductors, due to their band gaps and pristine surfaces, as well as lack of proper doping approach. To overcome this issue, several studies on specific cases of metal-TMD contacts have been reported recently: (1) Ti-MoS 2 and Au-MoS 2 top contacts [10] with the configuration shown in Fig. 3(a), which are only qualitatively studied by density-functional theory (DFT) in the absence of the treatment of van der Waals (vdW) interaction (which will be discussed later); 


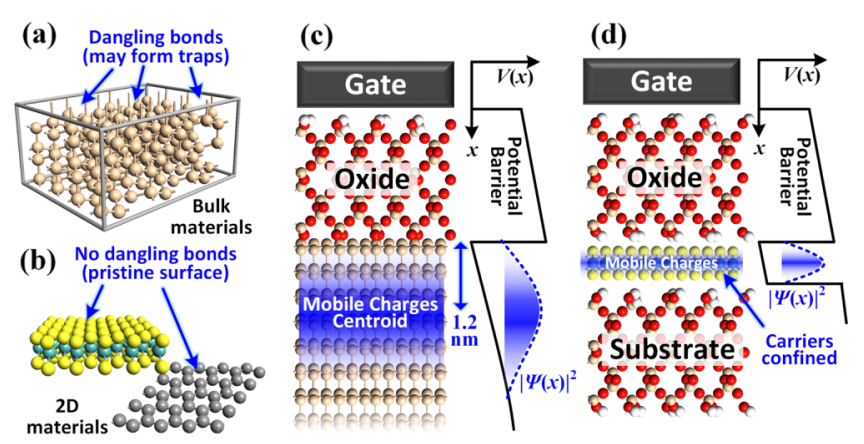

FIG. 1. Schematic illustrating advantages of 2D materials: surfaces of (a) 3D (bulk) and (b) 2D materials. The pristine interfaces (without out-of-plane dangling bonds) of 2D materials help reduce the interface traps. Mobile charge distribution in (c) 3D and (d) 2D crystals used as channel materials. $V(x)$ and $|\psi(x)|^{2}$ represent the potential and the probability density of the electronic charges, respectively. The carrier confinement effect in 2D materials leads to excellent gate electrostatics.

(2) $\mathrm{Sc}, \mathrm{Ni}$, and Au contacts to multilayer $\mathrm{MoS}_{2}$ [11]; (3) Ti contacts to multilayer $\mathrm{MoS}_{2}$ [12]; (4) Pd-WSe 2 contact [6]; (5) In-, Al-, and Ag-WSe 2 contact [7]; and (6) 2D compound metal contacts to $\mathrm{MoS}_{2}$ [13] studied by DFT. However, these works are not systematic and lack the rigorousness necessary for accurate analysis. On the other hand, the typical value of metal-mTMD contact resistance is still at least several $\mathrm{k} \Omega \mu \mathrm{m}$ and is usually $1-3$ decades higher than that of metal-silicon contacts in complementary metal oxide semiconductor technology (order of $0.1 \mathrm{k} \Omega \mu \mathrm{m}$ ) [14]. Such high contact resistances between metals and mTMDs significantly degrade the performance of TMD transistors [15]. Since there is currently no stable and reliable doping method to lower the contact resistance, it is highly desirable to explore suitable metals and contact configurations, which have the maximum potential to form low-resistance metal-mTMD contacts.

Hence, it is necessary to develop a comprehensive understanding of the nature of the electronic interface between metals and mTMDs, going beyond the analytical Schottky barrier (SB) theory. As mentioned above, currently such a comprehensive study of metal-mTMD contacts is still lacking. For example, diversity of metals, calculation of Schottky barrier height, and/or treatment of vdW force have not been considered simultaneously in all previous works. Moreover, all of the computational studies address only the properties of metal-mTMD top contacts [Fig. 3(a)] [10,13], while the edge contacts [Fig. 3(b)] have not been reported. Since 2D crystals are fundamentally different from 3D crystals in that the surface has no dangling bonds [Fig. 1(b)], one has to take advantage of the edges where there are dangling bonds for intimate chemical bonding for charge transfer.

In this work, we present a systematic study of the contacts between mTMDs (monolayer $\mathrm{MoS}_{2}$ and $\mathrm{WSe}_{2}$ ) and various metals (In, Ti, $\mathrm{Au}, \mathrm{Pd}, \mathrm{Mo}$, and $\mathrm{W}$ ) for different
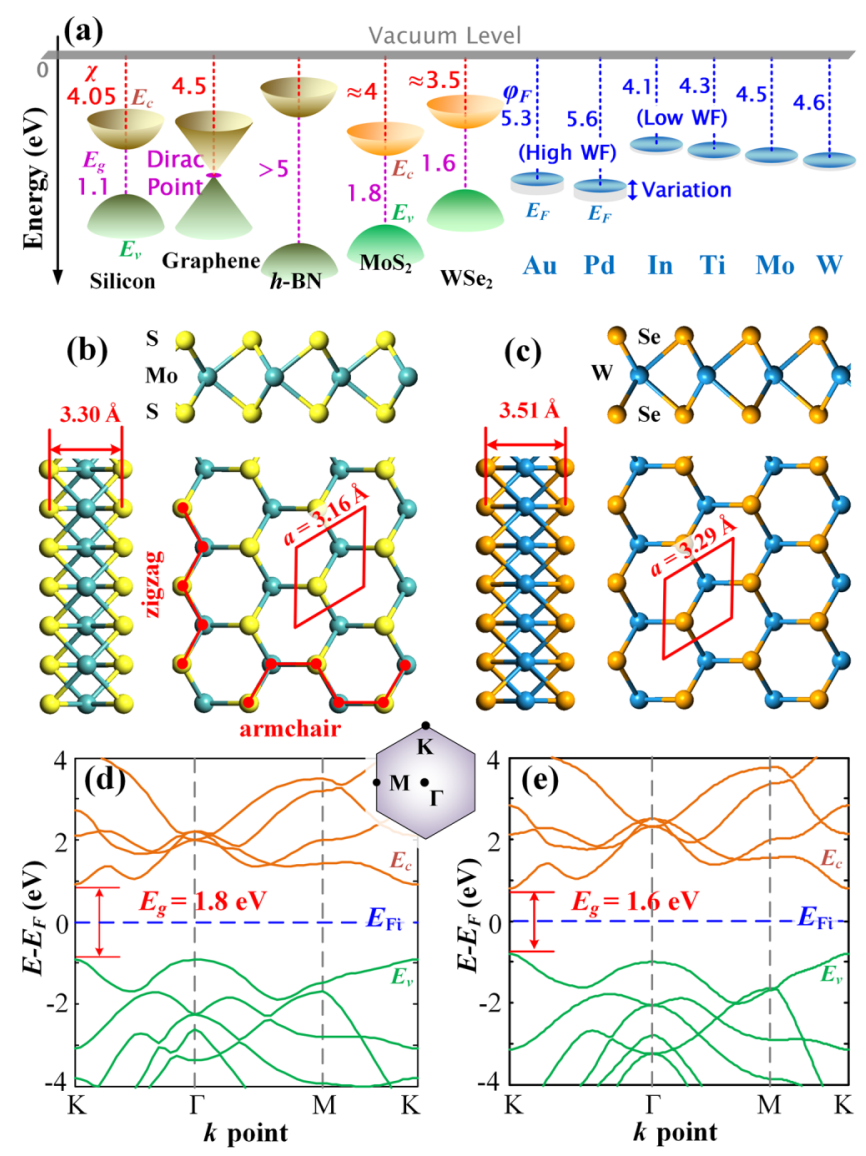

FIG. 2. (a) Band diagrams of silicon, some 2D materials, and selected contact metals. Graphene [1,2] has a zero-band-gap electronic dispersion at so-called Dirac points, while $h$-BN has a large $E_{g}(>5 \mathrm{eV})$ and can be used as an extremely thin dielectric layer [3]. $E_{c}, E_{v}$, and $E_{g}$ represent conduction band edge, valence band edge, and band gap, respectively. $\chi$ and $\phi_{F}$ represent electron affinities and metal work functions (WFs), respectively. (b),(c) Three views of lattice structures of (b) $\mathrm{MoS}_{2}$ and (c) $\mathrm{WSe}_{2}$. Red rhombuses represent primitive unit cells and $a$ is the lattice constant. Chirality is shown in (b) by armchair edge and zigzag edge. Lattice structures are relaxed using DFT with $8 \times 8 \times 1 k$ points sampled in the Brillouin zone (BZ). (d),(e) Energy dispersions of (d) $\mathrm{MoS}_{2}$ and (e) $\mathrm{WSe}_{2} . E_{\mathrm{Fi}}$ denotes the intrinsic Fermi level. Inset in (d) shows the first BZ of mTMDs containing the $M, K$, and $\Gamma$ points.

contact geometries (top and edge contacts) by DFT [16] considering the effect of $\mathrm{vdW}$ force. With the novel quantitative computational methodology presented in this work, we highlight and illustrate how to estimate the orbital overlaps and the Schottky or tunnel barriers to atomistic accuracy. It is shown that apart from choosing a proper work-function (WF) metal, the detailed physics of the

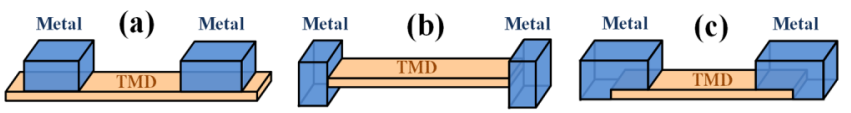

FIG. 3. Schematic of metal-mTMD (a) top contact, (b) edge contact, and (c) combined contact. 
interface between the metal and the mTMD layers plays an important role, which should be understood to achieve low contact resistances.

\section{METHODOLOGY}

The computational study of metal-mTMD contacts is further developed in four steps from the modeling and simulation framework used in our previous work [17], as listed in Fig. 4: (a) choosing metals (Sec. II A), (b) interface modeling (Sec. II B), (c) DFT calculations (Sec. II C), and (d) contact evaluation (Sec. III).

Compared to our previous work, in particular, the new methodology includes van der Waals interactions and employs the bond Mulliken population analysis of interfaces between $\mathrm{MoS}_{2}$ or $\mathrm{WSe}_{2}$ and various metal contacts, which is more robust, visual, and insightful and can guide experimental work. Moreover, band structure calculations are used to extract the Schottky barriers between metals and $\mathrm{MoS}_{2}$ or $\mathrm{WSe}_{2}$.

\section{A. Choosing metals}

In terms of the process robustness and electrical reliability, the bulk contact metals are still the main strategy for mTMDs compared to the 2D compound metals reported by Gan et al. [13]. Considering fundamental physical properties (melting point and electrical and thermal conductances) as well as chemical properties (stability and toxicity) of all metals, $\mathrm{Al}, \mathrm{Ti}, \mathrm{Cr}, \mathrm{Ni}, \mathrm{Cu}, \mathrm{Pd}, \mathrm{Ag}$, In, Pt, and Au are usually suitable as contact metals. However, the contact metals for mTMDs should have either low WF to achieve small $n$-type

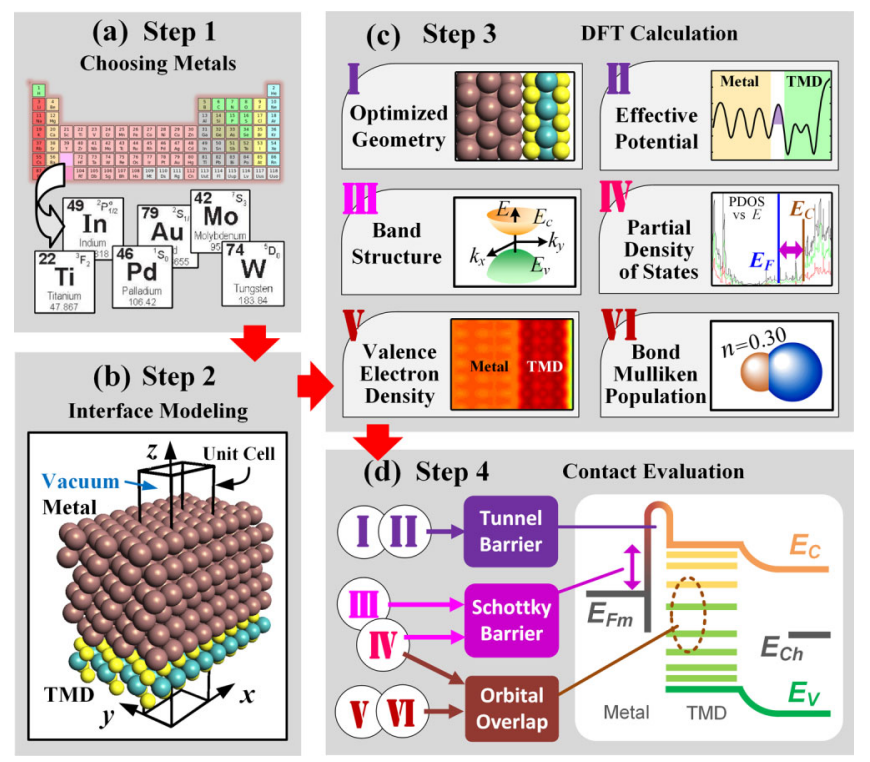

FIG. 4. Flow chart of the framework for metal-mTMD contact computational study in four steps: (a) choosing metals, (b) interface modeling, (c) DFT calculations, and (d) contact evaluation. $E_{\mathrm{vac}}$, $E_{c}, E_{v}$, and $E_{F}$ represent vacuum level, conduction band edge, valence band edge, and Fermi level, respectively. $E_{\mathrm{Fm}}$ and $E_{\mathrm{ch}}$ represent metal Fermi level and channel potential, respectively.
SBs or high WF to achieve small $p$-type SBs. Cr can be excluded because of its unsuitable WFs with respect to the mTMDs [18]. Furthermore, $\mathrm{Cr}$ and $\mathrm{Ni}$ can also be excluded due to the large lattice mismatches (percentage of lattice constant mismatch [19]) with mTMDs, because small lattice mismatches are favorable, which can maximize orbital overlaps (Fig. 5) [10]. Al is not a good contact metal for mTMDs because of the absence of $d$ orbitals, which can mix with the band-edge $d$ orbitals of Mo and $\mathrm{W}$ resulting in the better electron injection (Fig. 6) [7]. In addition, our experimental results show that $\mathrm{Al}$ and $\mathrm{Ni}$ form high-resistance contacts with mTMD [7].

Based on the above criteria, In, Ti, Au, and Pd are first chosen as the contact metals for this study in both top- and edge-contact configurations. Although Mo and $\mathrm{W}$ have neither high nor low WFs [Fig. 2(a)] (Mo: $4.5 \mathrm{eV}$; $\mathrm{W}: 4.6 \mathrm{eV}$ ) [18], they are the elements forming $\mathrm{MoS}_{2}$ and $\mathrm{WSe}_{2}$, respectively. Hence, Mo and $\mathrm{W}$ have great potential to form strong orbital overlaps with $\mathrm{MoS}_{2}$ and $\mathrm{WSe}_{2}$ by forming interface Mo-S and $\mathrm{W}-\mathrm{Se}$ bonds. Therefore, Mo and $\mathrm{W}$ are included in this study as well.

It is important to note that WF alone is not sufficient to form good contacts, as will be revealed in the subsequent sections in this paper. In Schottky theory, only an extremely high-WF or low-WF metal can form an Ohmic contact when Fermi level pinning is absent. However, in the absence of efficient doping methods for mTMDs, nearly no metal has such a high or low WF with respect to mTMDs. Hence, any top contact [Fig. 7(a)] will form one of the three types of Schottky contacts [Figs. 7(b)-7(d)]

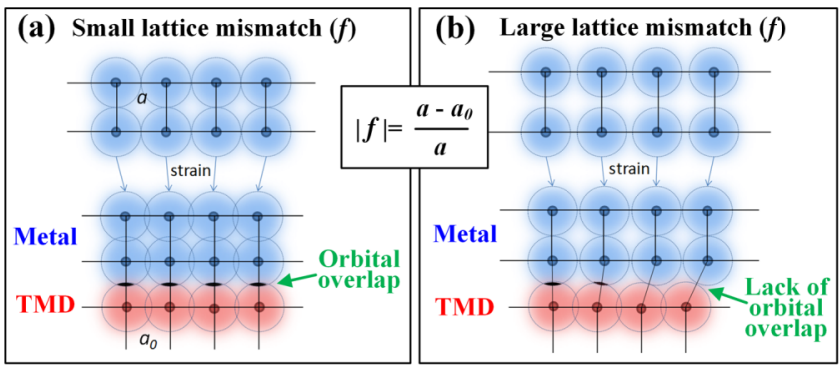

FIG. 5. Schematics showing the impact of lattice mismatches in metal-TMD contact. (a) Small lattice mismatch that maximizes the orbital overlaps between metal and TMD. (b) Large lattice mismatch that prevents maximizing the orbital overlaps.

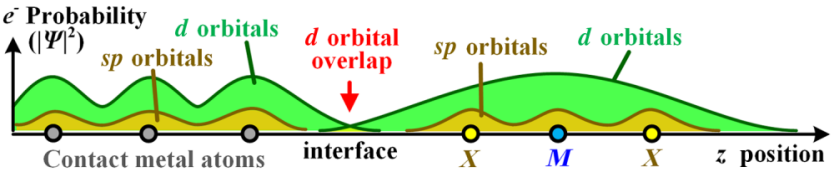

FIG. 6. Schematic showing the electron probabilities on different atomic orbitals of contact metal and mTMD $\left(M X_{2}\right)$. Metals with $d$ orbitals are preferred as contact metals due to the possible overlap with $d$ orbitals in mTMD, resulting in better electron injection. 

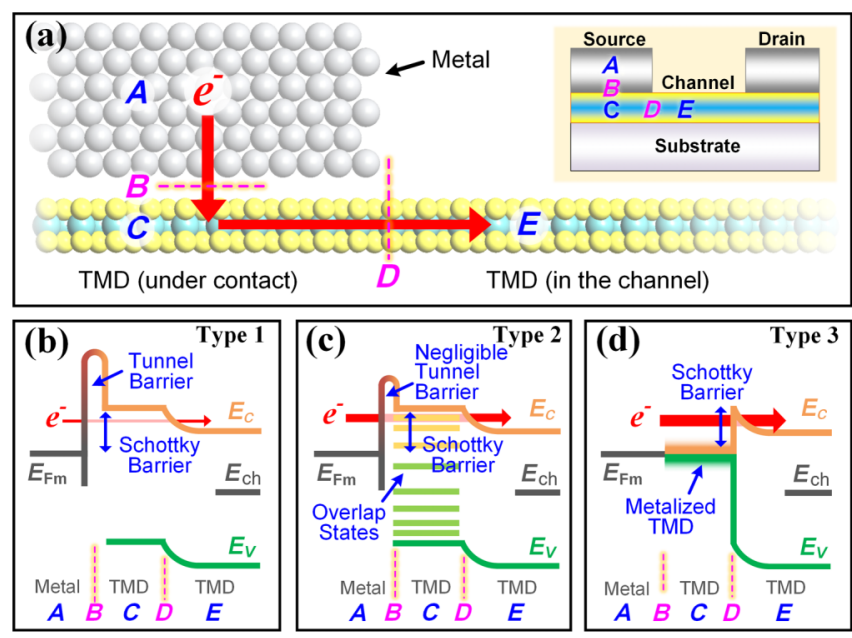

FIG. 7. (a) Schematic cross-sectional view of a typical metal$\mathrm{MoS}_{2}$ contact ( $n$-type top contact). $A, C$, and $E$ denote the three regions while $B$ and $D$ are the two interfaces separating them. Red arrows show the pathway $(A \rightarrow B \rightarrow C \rightarrow D \rightarrow E)$ of electron injection from contact metal $(A)$ to the $\mathrm{MoS}_{2}$ channel $(E)$. The inset shows the source and drain contacts and the channel region in a typical backgated FET. (b)-(d) The three possible band diagrams of (a): metal contacts with (b) very weak bonding, (c) medium bonding, and (d) strong bonding. $E_{c}, E_{v}$, $E_{\mathrm{Fm}}$, and $E_{\mathrm{ch}}$ represent conduction band edge, valence band edge, metal Fermi level, and channel potential, respectively.

(or their corresponding $p$-type contacts): type 1, metals with very weak adhesion with mTMDs; type 2 , medium adhesion; and type 3, strong adhesion. For type 3, as will be revealed later, these metals can form interface covalent bonds with mTMDs [at interface $B$ in Fig. 7(a)], which strongly perturbs the band structure of mTMD and results in vanishing of the mTMD band gap [between $B$ and $D$ in Fig. 7(d)] under metal. Therefore, the mTMD is metalized and the Schottky barrier under the metal [at interface $B$ in Fig. 7(d)] vanishes, which leads to an Ohmic contact under the metal (at $B$ ) and a thinner Schottky barrier at the sourcedrain channel junction (interface $D$ ). Although the semiconducting properties of mTMD under the contact metal are distorted, the channel region is not affected. Hence, this kind of contact is preferred for mTMD devices.

\section{B. Interface modeling}

As shown in Fig. 4(b), metal-mTMD contact regions are modeled, which are periodic in the $x$ and $y$ directions and separated by vacuum in the $z$ direction. For top contacts, as shown in Figs. 8 and 9, the contact region contains an intrinsic mTMD monolayer and the close-packed surfaces of a metal [In(101), Ti(001), Au(111), Pd(111), Mo(001), or W(001)] extending to the sixth layer. These orientations are the most probable to be found in experiments. In this work, to emulate the effect of upper layers in modeling, the third to sixth layers of metals from the interface are set as constraints (atoms with fixed locations), as shown in
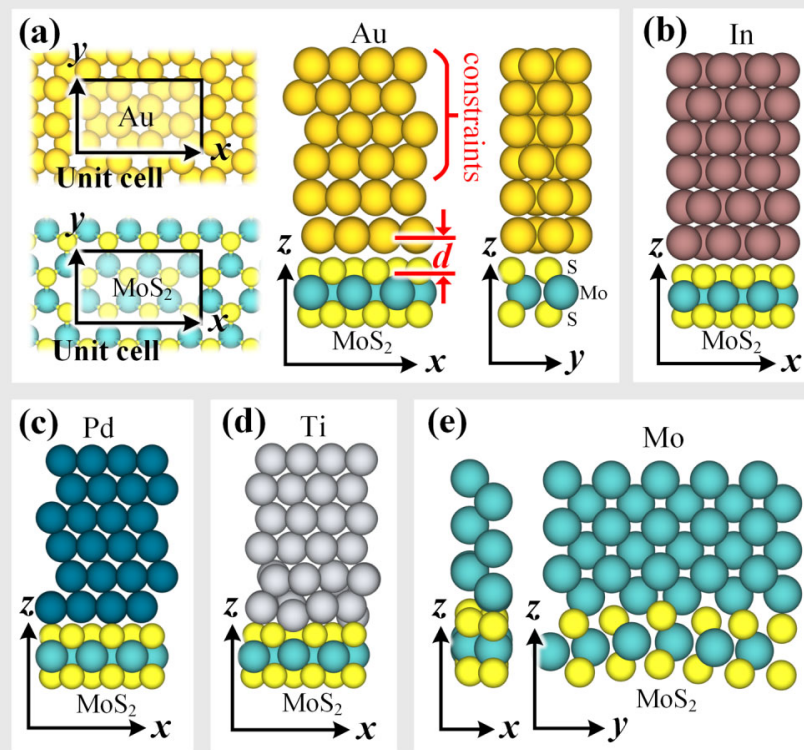

(e)

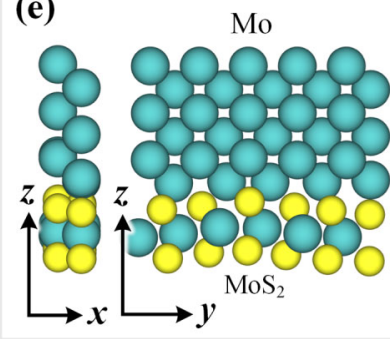

FIG. 8. Optimized geometries of top contacts to $\mathrm{MoS}_{2}$ : (a) $\mathrm{Au}-\mathrm{MoS}_{2}$ (in different views), (b) In- $\mathrm{MoS}_{2}$, (c) $\mathrm{Pd}-\mathrm{MoS}_{2}$, (d) $\mathrm{Ti}-\mathrm{MoS}_{2}$, (e) $\mathrm{Mo}-\mathrm{MoS}_{2}$ (in different views). $d$ is defined as the physical separation (the $z$ component of the nearest core-tocore distance between the metal atoms and the chalcogenide atoms). Radii of the atomic spheres shown in (a)-(e) are fixed to the covalent radius of the elements, which is a measure of the size of an atom that forms part of one covalent bond. Hence, the touching of atomic spheres indicates the formation of covalent bonds [e.g., the Ti-S bond in (d)].

Fig. 8(a). mTMD as well as first to second metal layers are allowed to relax. Although in real situations the contact metals consist of many layers, we restrict the simulation to only six layers of metal atoms because the obtained results do not change appreciably beyond this thickness [17].

There are many ways to terminate the mTMD layers at the edges depending on the contact orientations. In particular, armchair termination of $\mathrm{MoS}_{2}$ leads to semiconducting behavior, and its electronic properties are weakly dependent on the ribbon width, while the entire zigzag $\mathrm{MoS}_{2}$ ribbon exhibits metallic behavior [20]. Hence,

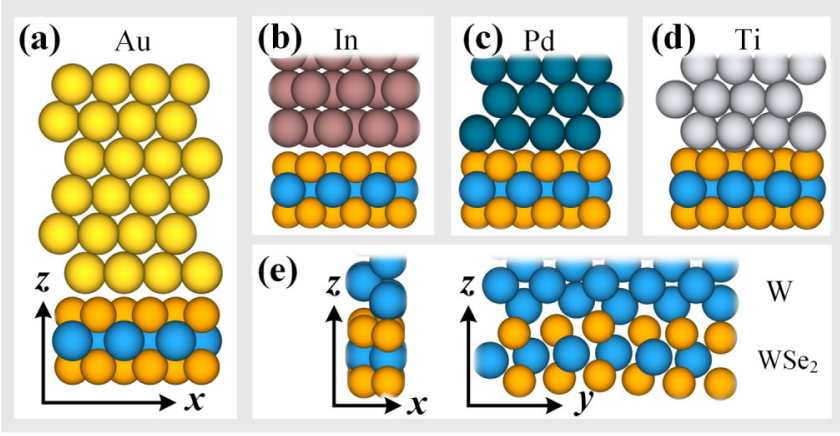

FIG. 9. Optimized geometries of top contacts to $\mathrm{WSe}_{2}$ : (a) $\mathrm{Au}-\mathrm{WSe}_{2}$, (b) In-WSe, (c) $\mathrm{Pd}-\mathrm{WSe}_{2}$, (d) $\mathrm{Ti}_{2}-\mathrm{WSe}_{2}$, (e) $\mathrm{W}-\mathrm{WSe}_{2}$ (in different views). 
to preserve the semiconducting properties of mTMDs as much as possible, which is also the worst case for electron injection due to the band gap, we choose the armchair edges to form interfaces (which will be shown in Fig. 18). Four out of six mTMD unit cells on the left are set as constraints [Fig. 18(a)] to emulate the effect of a long mTMD layer on the left (same as the configuration for metal-graphene edge contacts [21]), while all other atoms (including four layers of metal atoms) are allowed to relax.

\section{DFT calculations}

The first-principles calculations are performed by DFT. Using DFT approaches, the properties of a many-electron system can be determined in the form of a spatially dependent electron density [16], which makes it possible to incorporate quantum mechanical effects in the density function ( 3 degrees of freedom) rather than through manybody wave functions $(3 \times N$ degrees of freedom).

The DFT approach employed in this work is the KohnSham DFT [22], where the problem of interacting electrons in a static external potential is reduced to a problem of noninteracting electrons moving in an effective potential. The effective potential includes the external potential and the effects of the Coulomb interactions between the electrons, which is described by the exchange and correlation interactions.

Though, Kohn-Sham DFT has significant computational advantages over other $a b$ initio methods, it is well known that the widely used exchange and correlation interactions of the generalized gradient approximation (GGA) [23] or the local density approximation (LDA) [24] do not give accurate results for band gaps for some semiconductor materials. To ensure that the simulations are accurate, we first investigate the band structures of monolayers $\mathrm{MoS}_{2}$ and $\mathrm{WSe}_{2}$ calculated with either the LDA exchange correlation or the Perdew-Burke-Ernzerhof variant [23] of GGA (PBE GGA), together with either the double- $\zeta$ polarized (DZP) basis set or the Hartwigsen-GoedeckerHutter (HGH) basis set for expanding the electronic density. The results show that LDA with DZP or HGH and GGA with HGH give a direct band gap of $1.8 \mathrm{eV}$ for the monolayer $\mathrm{MoS}_{2}$, which is consistent with results from experiments [25], while for the monolayer $\mathrm{WSe}_{2}$, only LDA gives a direct band gap of $1.6 \mathrm{eV}$ that is consistent with the results obtained from both theory and experiments [26]. A more detailed evaluation is included in the Appendix. Hence, LDA with HGH is chosen for all DFT simulations in this work.

Though accurate descriptions of covalent and ionic chemical bonds can be achieved with the settings as discussed above, they may fail to reproduce nonlocal dispersive forces, in particular, van der Waals forces, which are important in weakly bonded systems such as interfaces of two materials bonded with vdW, multilayer 2D materials, molecular crystals, and organic compounds [27].
As discussed in Sec. I, this problem has not been addressed in previous works on interfaces with mTMDs $[10,17]$, so the accuracy of the results from those works may be limited. Hence, the interfaces with 2D materials clearly require the development of new DFT methods designed to overcome this problem $[28,29]$, by alterations to the functional or by the inclusion of additive terms, as shown for graphene [27]. Pragmatic methods to address this problem have been provided by approaches such as DFT-D or DFT-D2 [30-32] and vdW-DF [33-35]. In DFT-D or DFT-D2 approaches, a semiempirical dispersion potential is added to the conventional Kohn-Sham DFT energy, where the potential is described via a simple pairwise force field and is optimized for popular DFT functionals.

Hence, in this work, DFT-D2 is adopted due to its higher accuracy, broader range of applicability, and lesser empiricism [32]. The calculations were performed using the Atomistix ToolKit (ATK) [36]. $8 \times 16 \times 1 k$ points were sampled in the Brillouin zone (BZ) of the top-contact region, while the edge-contact BZ was meshed by $8 \times 8 \times 1 k$ points. The density mesh cutoff was $200 \mathrm{Ry}$ and the maximum force was $0.05 \mathrm{eV} / \AA$ f for geometry optimizations.

\section{CONTACT EVALUATION}

Three major criteria (tunnel barrier, Schottky barrier, and orbital overlap) are analyzed to evaluate the electron injection efficiency of contacts as shown in Fig. 4(d), since they can sufficiently capture the essential interface characteristics of metal TMDs that determine their electrical behavior.

The first criterion - tunnel barrier - can be inferred from blocks I and II of Fig. 4 (the optimized geometry and the effective potential) calculated using DFT. A narrow and low tunnel barrier at the metal-mTMD interface can increase the electron injection efficiency.

Block I, optimized geometry, is the relaxed structure with minimum total energy, which reflects the nature of ideal interfaces theoretically. Physical separations (d) [defined in Fig. 8(a)] are measured from optimized geometries. $d$ is directly related to the width $(\leq d)$ of the tunnel barrier between metal and mTMD.

In block II, the effective potential ( $\left.V_{\text {eff }}\right)$ of an electron represents its interaction with other electrons and the external electrostatic field. $V_{\text {eff }}$ is calculated by $V_{\text {eff }}(n)=$ $V_{H}(n)+V_{\text {xc }}(n)+V_{\text {ext }}$, where $V_{H}(n)$ is the Hartree potential due to the mean-field electrostatic interaction, $V_{\mathrm{xc}}(n)$ is the exchange-correlation potential caused by the quantum mechanical nature of the electrons, and $V_{\text {ext }}$ represents other electrostatic interactions in the system. The tunnel barrier height can be characterized by the peak of $V_{\text {eff }}$ at the interface, which is noted as effective tunnel barrier height $\left(\Phi_{\mathrm{TB}, \text { eff }}\right)$ [defined in Fig. 10(a)].

The Schottky barrier can be determined by blocks III and IV (the band structure and the partial density of states). 


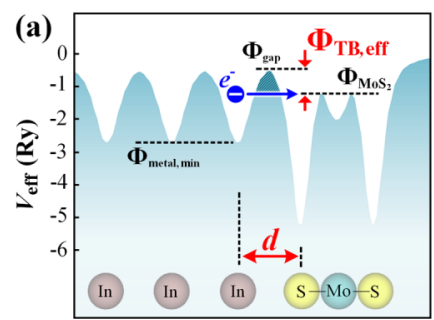

Position on $z$ axis

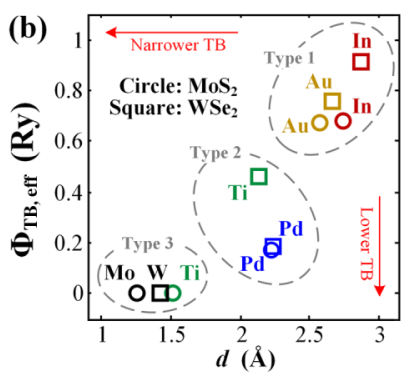

FIG. 10. Evaluation of the tunnel barriers at the contacts. (a) Plot of minimum effective potential $\left(V_{\text {eff }}\right)$ versus $z$ position for In-MoS 2 top contact. $\Phi_{\mathrm{MoS}_{2}}$ is the $V_{\text {eff }}$ of the Mo-S bond orbitals and thereby the effective tunnel barrier height $\left(\Phi_{\mathrm{TB}, \text { eff }}\right)$ is defined as the minimum barrier height that an electron from the metal has to overcome if it has the same potential energy as $\Phi_{\mathrm{MoS}_{2}}$. Hence, $\Phi_{\mathrm{TB} \text {,eff }}$ can be calculated as the $V_{\text {eff }}$ difference between the vdW gap $\left(\Phi_{\text {gap }}\right)$ and $\operatorname{MoS}_{2}\left(\Phi_{\mathrm{MoS}_{2}}\right)$. $\Phi_{\text {metal,min }}$ denotes the minimum $V_{\text {eff }}$ that an electron can have in the metal. It is worth noting that in some metals (such as Au) $\Phi_{\text {metal,min }}$ can be higher than $\Phi_{\mathrm{MoS}_{2}}$ (thus, electron energy is always higher than that of Mo-S bond orbitals), in which case $\Phi_{\mathrm{TB}, \text { eff }}$ is calculated as $\Phi_{\mathrm{TB}, \text { eff }}=\Phi_{\text {gap }}-\Phi_{\text {metal,min }}$. $\Phi_{\mathrm{TB}, \text { eff }}$ vanishes to zero when $\Phi_{\text {metal,min }}$ or $\Phi_{\mathrm{MoS}_{2}}$ is higher than $\Phi_{\text {gap }} . d$ is defined as the physical separation (the $z$ component of the nearest core-to-core distance between the metal atoms and the chalcogenide atoms). (b) $\Phi_{\mathrm{TB}, \text { eff }}$ versus $d$ plot for various top contacts.

In block III, the band structure (or energy dispersion) can be calculated for the metal-mTMD contact system. By comparing the original band structure of $\mathrm{MoS}_{2}$ without contact and the new band structure after contact, the shift of Fermi level $\left(E_{F}\right)$ can be identified, as can the Schottky barrier.

In block IV, the partial density of states (partial DOS or PDOS) is the density of states on specified atoms and orbitals. The Schottky barrier can also be measured by the energy difference between conduction or valence band edge $\left(E_{c}\right.$ or $\left.E_{v}\right)$ of mTMD and $E_{F}$ of the metal-mTMD contact system.

As shown in Figs. 4(c) and 4(d), (electron) orbital overlap (in other words, bond formation) is evaluated by blocks IV, V, and VI in Fig. 4(c). By comparing the PDOS on mTMDs before and after contact formation, overlap states can be found, the density of which indicates the strength of orbital overlaps in the energy domain.

In block $\mathrm{V}$, valence electron density (at the interfaces) indicates the strength of overlapped electron orbitals in the real space. High (valence) electron density at the interfaces allows sufficient injection of charge into the mTMD layer [10].

In block VI, bond Mulliken population is the overlap population of electrons for pairs of atomic orbitals [37]. This result gives a visual and quantitative evaluation of the orbital overlap. Bond Mulliken populations $n$ have a typical range of $0 \leq n \leq 1$, where $n=0,0<n<1$, and $n=1$ indicate typical ionic bond, covalent bond, and the strongest covalent bond, respectively. The population indicates the strength of the covalent bond, or in other words, the strength of the orbital overlaps in terms of shared electron numbers. For example, the Mo-S (W-Se) covalent bond in $\mathrm{MoS}_{2}\left(\mathrm{WSe}_{2}\right)$ has a population of $0.53(0.50)$.

\section{A. Tunnel barriers of top contacts}

The tunnel barrier between a metal and TMD is characterized by its width and height, which are evaluated by the physical separation $(d$, measured from the optimized geometry) and effective tunnel barrier height $\left(\Phi_{\mathrm{TB}, \text { eff }}\right)$, respectively.

To evaluate the tunnel barrier widths, optimized geometries of top contacts are simulated and shown in Figs. 8 and 9 . The physical separations $(d)$ between the metal and mTMD atoms are defined as shown in Figs. 8(a) and 10(a). For all of the top contacts, $d$ is calculated and plotted in Fig. 10(b). Because of the smaller atomic sizes of $\mathrm{MoS}_{2}$ compared to that of $\mathrm{WSe}_{2}$, top contacts to $\mathrm{MoS}_{2}$ have smaller physical separations. For low-WF metal (In, Ti) top contacts to mTMD, Ti gives much smaller $d(1.51 \AA$ to $\mathrm{MoS}_{2}$ and $2.13 \AA$ to $\left.\mathrm{WSe}_{2}\right)$ than that of In $\left(2.58 \AA\right.$ to $\mathrm{MoS}_{2}$ and $2.67 \AA$ to $\mathrm{WSe}_{2}$ ). While for high-WF metal (Au, Pd) top contacts, Pd-mTMD top contacts give smaller physical separations than Au-mTMD top contacts, as shown in Fig. 10(b).

Mo and W top contacts to mTMD are evaluated in the same manner. However, $d$ values of $\mathrm{Mo}-\mathrm{MoS}_{2}$ and $\mathrm{W}-\mathrm{WSe}_{2}$ top contacts are extremely small (1.25-1.42 $\AA$ ) compared to all of the other metal-mTMD top contacts (1.51-2.87 ̊). Those small physical separations may lead to extremely thin tunnel barriers and strong orbital overlaps. Moreover, it can be clearly seen from Fig. 8(e) that atoms in mTMDs are dragged by Mo (W) atoms to form Mo-S (W-Se) interface bonds, resulting in the breaking of mTMD periodicity by deformation. Hence, strong disturbing of mTMD band structures [which will be shown later in Fig. 13(c)] can be expected.

To evaluate the tunnel barrier heights, effective potentials $\left(V_{\text {eff }}\right)$ are calculated. The minimum effective potential $\left(V_{\text {eff }}\right)$ along the $z$ direction for the In-MoS 2 top contact is shown in Fig. 10(a) as an example. As illustrated by Fig. 10(a), the effective tunnel barrier height $\left(\Phi_{\mathrm{TB}, \text { eff }}\right)$ can be measured from $V_{\text {eff }}$ and $\Phi_{\mathrm{TB} \text {,eff }}$ of each contact, as plotted in Fig. 10(b). Similarly, top contacts to $\mathrm{MoS}_{2}$ have lower tunnel barriers than those of top contacts to $\mathrm{WSe}_{2}$. According to the results, Au- and In-mTMD contacts have high $\Phi_{\mathrm{TB}, \text { eff }}(0.67-0.92 \mathrm{eV})$, while for Mo- and Ti- $\mathrm{MoS}_{2}$ and $\mathrm{W}-\mathrm{WSe}_{2}$ top contacts there is nearly no barrier $\left(\Phi_{\mathrm{TB}, \mathrm{eff}}=0 \mathrm{eV}\right)$ at the interface, indicating high electron injection efficiency and thus low contact resistance.

Based on the evaluation of the tunnel barriers, the types of contacts [Figs. 7(b)-7(d)] can be preliminarily predicted, as shown by type 1, type 2, and type 3 in Fig. 10(b). 


\section{B. Schottky barriers of top contacts}

To further evaluate the top contacts and find the SBs, PDOS are calculated and shown in Figs. 11(b)-11(f), 12(b)-12(f), and compared to mTMD without contacts [Figs. 11(a) and 12(a)].

In Figs. 11(b), 11(c), 11(e), and 11(f) [Figs. 12(b), 12(c), 12(e), and 12(f)], the position of $E_{F}$ is shifted towards the original conduction band $\left(E_{c}\right)$ indicating that $\mathrm{MoS}_{2}\left(\mathrm{WSe}_{2}\right)$ is doped $n$ type by Au, In, Ti, or Mo (W). The $E_{F}$ of the $\mathrm{Pd}-\mathrm{MoS}_{2}$ top contacts lie at the middle of the $\mathrm{MoS}_{2}$ band gap [Fig. 11(d)], indicating that $\mathrm{MoS}_{2}$ is still nearly intrinsic with the Au top contact. In contrast, $E_{F}$ is close to the original $E_{v}$ of monolayer $\mathrm{WSe}_{2}$. Hence, the $\mathrm{Pd}-\mathrm{WSe}_{2}$ top contact is shown to be a $p$-type contact [Fig. 12(d)]. This is the only $p$-type top contact found in this study, in agreement with experimental results on Pd-contacted $\mathrm{MoS}_{2}$ devices [6].

By measuring the energy difference between $E_{F}$ and the original $E_{c}$, the Schottky barrier height $\left(\Phi_{\mathrm{SB}}\right)$ for each contact can be estimated, as indicated in Figs. 11(b), 11(c) [or Figs. 12(b), 12(c)]. However, more precise results can be achieved by the analysis of band structures. In Fig. 13, the band structures of Au-, Ti-, and Mo-MoS 2 systems are plotted (in gray). The original band structure of $\mathrm{MoS}_{2}$ without contact is also plotted for reference (red curves), which is superimposed on the new band structure (gray curves) such that old and new subbands align. $\Phi_{\mathrm{SB}}$ in each plot is then measured accordingly. For example, in Au- and Ti-MoS 2 top contacts, the Schottky Barriers are 0.62 and
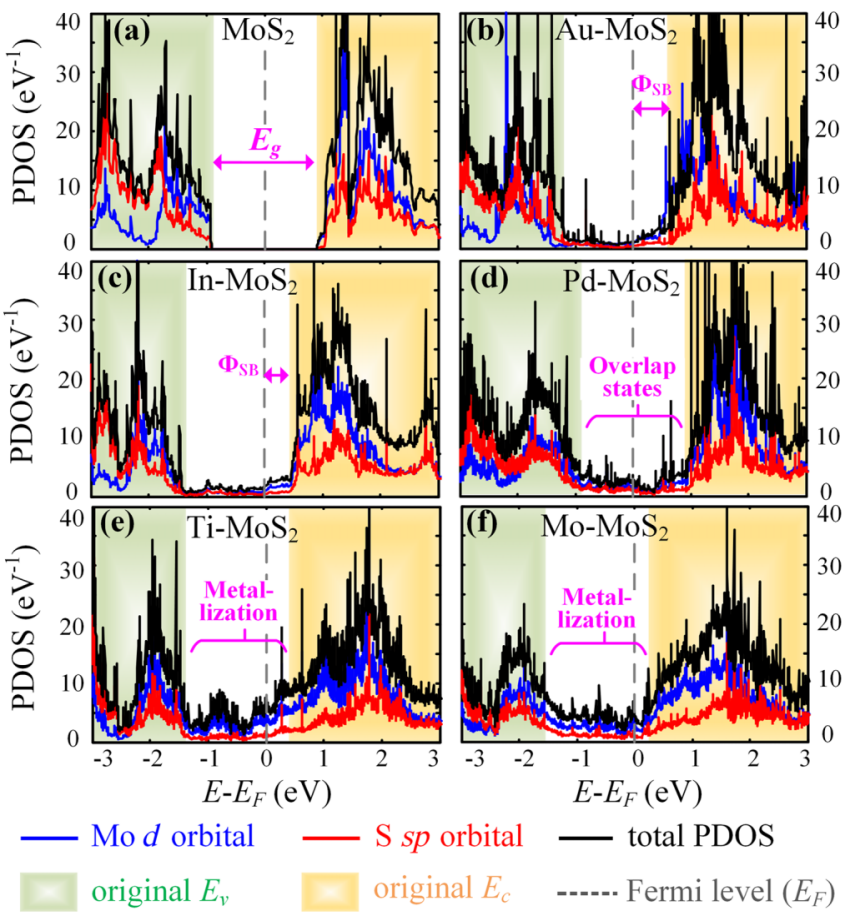

FIG. 11. Partial density of states (PDOS) [DOS on specified atoms and orbitals, for example, Mo- $d$ ( $d$ orbital on Mo)] of top contacts to $\mathrm{MoS}_{2}$ : (a) only $\mathrm{MoS}_{2}$, (b) $\mathrm{Au}-\mathrm{MoS}_{2}$, (c) In-MoS (d) $\mathrm{Pd}-\mathrm{MoS}_{2}$, (e) Ti-MoS 2 , (f) Mo-MoS $2 . E_{F}$ denotes Fermi level.
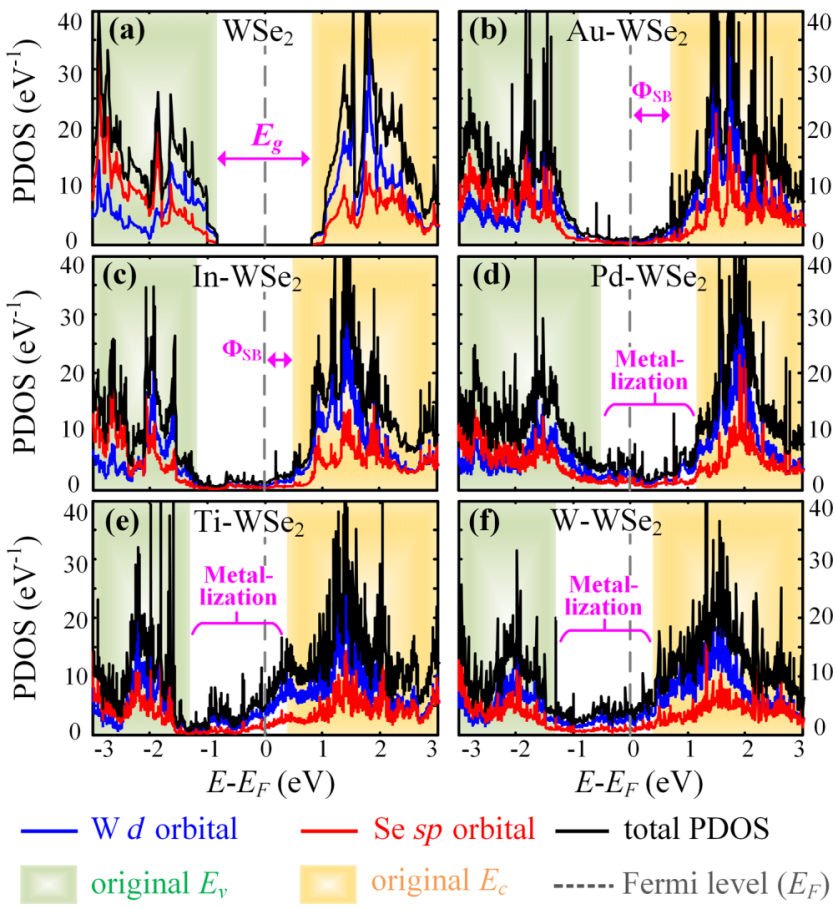

FIG. 12. PDOS of top contacts to $\mathrm{WSe}_{2}$ : (a) only $\mathrm{WSe}_{2}$, (b) $\mathrm{Au}-\mathrm{WSe}_{2}$, (c) In-WSe, (d) $\mathrm{Pd}-\mathrm{WSe}_{2}$, (e) $\mathrm{Ti}_{-}-\mathrm{WSe}_{2}$, (f) $\mathrm{W}-\mathrm{WSe}_{2} . E_{F}$ denotes Fermi level.
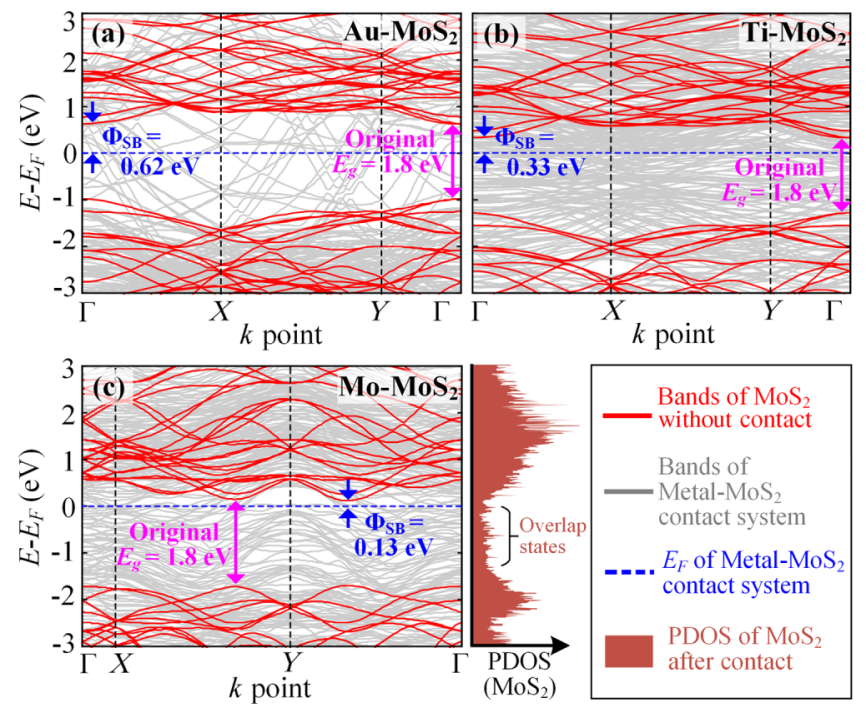

FIG. 13. Band structures of (a) Au-MoS 2 system, (b) Ti-MoS system, and (c) Mo-MoS 2 system. The original band structure of $\mathrm{MoS}_{2}$ without contact is also plotted for reference (red curves), which is superimposed on the new band structure (gray curves) such that old and new subbands align. The Schottky barrier $\left(\Phi_{\mathrm{SB}}\right)$ is marked in blue. Note that the symmetric points $(\Gamma, X$, and $Y$ ) are different between (a), (b), and (c) due to different dimensions of the unit cells. PDOS of $\mathrm{MoS}_{2}$ after contact by Mo is also shown in (c). 

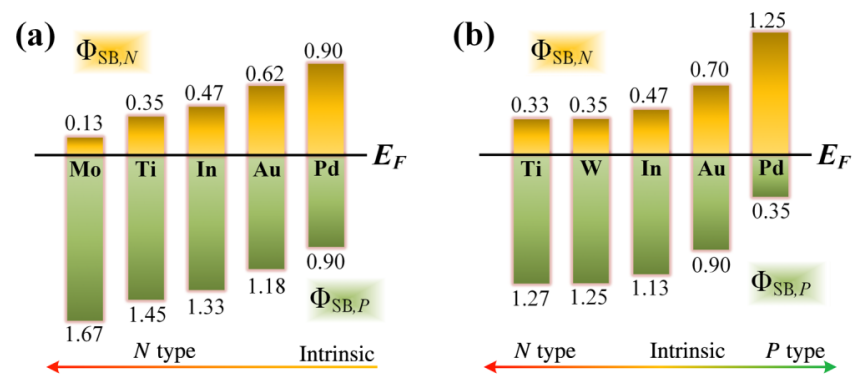

FIG. 14. $\Phi_{\mathrm{SB}}$ of all of the (a) $\mathrm{MoS}_{2}$ top contacts and (b) $\mathrm{WSe}_{2}$ top contacts. $\Phi_{\mathrm{SB}, N}\left(\Phi_{\mathrm{SB}, P}\right)$ denotes $n$ type ( $p$ type) $\mathrm{SB}$ for electrons (holes).

$0.33 \mathrm{eV}$, respectively, from DFT simulation as shown in Figs. 13(a) and 13(b). Using this approach, $\Phi_{\mathrm{SB}}$ for all of the top contacts is calculated and listed in Fig. 14. According to our recent experimental work (under review), the extracted Schottky barrier between Ti and monolayer $\mathrm{MoS}_{2}$ varies between 0.3 and $0.35 \mathrm{eV}$ measured from 6 monolayer devices, which is in agreement with the simulation results $(0.33 \mathrm{eV})$.

Moreover, despite the high WF $(4.5 \mathrm{eV})$ of Mo [Fig. 2 (a)], the Fermi level is pinned at only $0.13 \mathrm{eV}$ below the original $E_{c}$ of intrinsic monolayer $\mathrm{MoS}_{2}$ [Fig. 2(c)], indicating a Schottky barrier of $0.13 \mathrm{eV}$ at interface $D$ in Fig. 7(d). This Schottky barrier height is much lower than that of the Ti-MoS $\mathrm{Mop}_{2}$ contact $[0.33 \mathrm{eV}$, as shown in Fig. 13(b)], although Ti has a smaller WF that is also closer to the electron affinity of $\mathrm{MoS}_{2}$ [4.3 eV, Fig. 2(a)]. This also indicates that the properties of contacts to $2 \mathrm{D}$ materials cannot be intuitively predicted by WF values.

It is instructive to note that such an abnormal Fermi level pinning at the $\mathrm{Mo}-\mathrm{MoS}_{2}$ interface is not caused by interface traps, which may consume incoming carriers, because of the absence of dangling bonds (interface traps) in $\mathrm{MoS}_{2}$. Since the $\mathrm{MoS}_{2}$ is monolayer, its properties can be easily distorted by the strong orbital overlaps (covalent bonds), which create overlap states. Thus, the electronic properties of the $\mathrm{MoS}_{2}$ under the contact change. In other words, the compound (Mo- $\mathrm{MoS}_{2}$ alloy) at the interface can be expected to be a new material, which has a much lower WF compared to that of the unalloyed $\mathrm{MoS}_{2}$ in the channel region [Fig. 7(a)]. Hence, the unalloyed $\mathrm{MoS}_{2}$ (near the contacts) is $n$-type doped as if it is contacted to a low-WF metal. This phenomenon has also been confirmed in the simulation of the Mo contact on multilayer $\mathrm{MoS}_{2}$ [38].

On the other hand, it is also important to note that the study of the unusual Fermi level pinning at the metal-TMD interface requires careful treatment of the $\mathrm{vdW}$ interaction between them, which is usually missing in studies of $2 \mathrm{D}$ interfaces. Using pure LDA, no reliable predictions can be made due to its limitations in handling vdW interactions [28]. In particular, LDA does not correctly reproduce the interlayer binding energies [29]. DFT-D functionals give the closest interlayer binding energy results for layered
$\mathrm{MoS}_{2}$ and $\mathrm{WSe}_{2}$ with respect to the comprehensively and experimentally tested random-phase approximation method, compared with LDA, PBE GGA, and vdW-DF [28]. Also, considering its lower computational demands, DFT-D is more useful for describing vdW interaction. Hence, the adoption of the DFT-D2 (newer version of DFT-D) functional in this work is highly necessary and suitable, and thus the results are more reliable.

\section{Orbital overlap of top contacts}

Because of the lack of orbital overlaps, Au- and In-mTMD top contacts are typical Schottky contacts [type 1 in Fig. 7(b)]. In contrast, for $\mathrm{Pd}-\mathrm{MoS}_{2}$ top contacts [Fig. 11(d)], overlap states can be found in the original band gap of $\mathrm{MoS}_{2}$. This indicates that the $\mathrm{Pd}-\mathrm{MoS}_{2}$ top contact is type 2 [Fig. 7(c)]. As illustrated in Fig. 7(c), these overlap states contribute to the electron or hole injection from the metal.

As shown earlier, the overlap of $\mathrm{Ti}$ and $\mathrm{S}$ atomic spheres can be clearly observed from the optimized geometry of the Ti-MoS $\mathrm{S}_{2}$ top contact Fig. 8(d), which indicates the high possibility of covalent bond formation between $\mathrm{Ti}$ and $\mathrm{MoS}_{2}$. This is proved by Fig. 11(e), where the high PDOS spreads all over the original band gap, which represents the overlap states corresponding to the covalent bonds. Hence, Ti-MoS 2 has an Ohmic interface [at $B$ in Fig. 7(d)], where the band gap vanishes and the $\mathrm{MoS}_{2}$ region under the contact metal [Fig. 7(a)] is metallized. Similar results are also found in $\mathrm{Mo}-\mathrm{MoS}_{2}$, Ti-WSe 2 , Pd-WSe ${ }_{2}$, and W-WSe top contacts. Hence, although Mo and $\mathrm{W}$ do not have suitable WFs for $\mathrm{MoS}_{2}$ and $\mathrm{WSe}_{2}$, respectively, both Mo- $\mathrm{MoS}_{2}$ and W-WSe $\mathrm{W}_{2}$ top contacts have Ohmic interfaces [B in Fig. 7(d)]. These results can be found only by atomic level modeling (i.e., DFT) and cannot be inferred intuitively from analytical Schottky barrier theory.

As shown in Figs. 13(b) and 13(c), most of the original $\mathrm{MoS}_{2}$ bands (red) are disturbed by Ti or Mo contact, forming new bands (gray) that extend into the original band gap, which correspond to the covalent bands with overlap states, while most of the $\mathrm{MoS}_{2}$ bands in the Au-MoS 2 system remain the same as in pure $\mathrm{MoS}_{2}$ [Fig. 13(a)], indicating the lack of orbital overlaps. It is important to note that electrons on the overlap states in the Ti- and Mo-MoS 2 systems are not localized, so that the metal will not degrade the conductivity of $\mathrm{MoS}_{2}$ under the contact. This can be confirmed by the shapes of the energy bands of the Ti- and Mo-MoS 2 system (gray curves) in Figs. 13(b) and 13(c), where most of the energy bands have high enough curvature (indicating small effective mass) for efficient carrier transport.

Valence electron densities of top contacts are calculated and shown in Fig. 15. The minimum values of the $x-y$ plane averages $\left(\rho_{m}\right)$ at the interfaces are measured and marked on the curves or contours. The Au and In contacts give relatively lower $\rho_{m}$, indicating weak adhesion and, thus, weak orbital overlaps. For Pd top contacts, the corresponding values are 


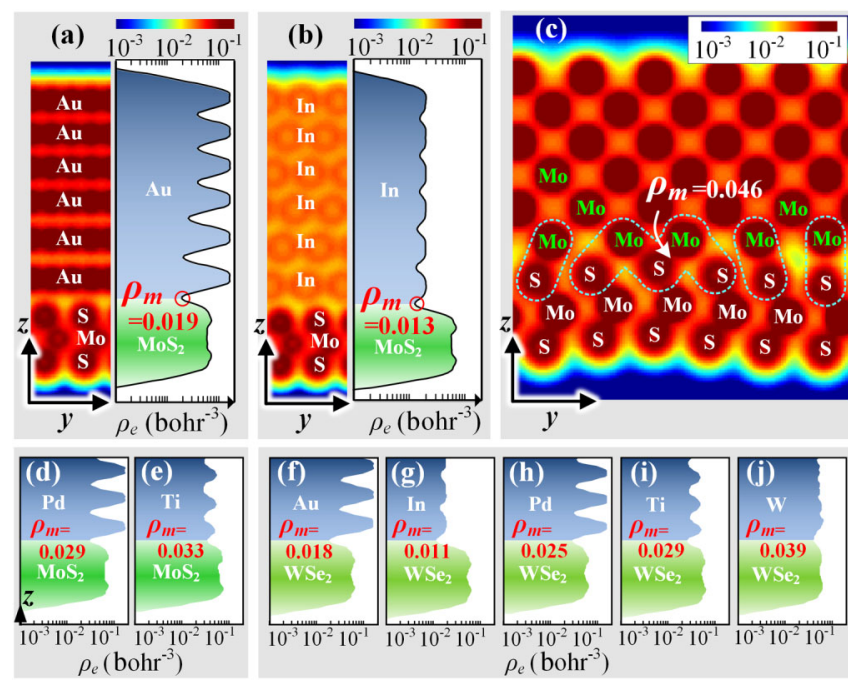

FIG. 15. Valence electron densities $\left(\mathrm{bohr}^{-3}\right)$ of metal-mTMD top contacts: (a) Au-MoS 2 , (b) In-MoS 2 , (c) $\mathrm{Mo}-\mathrm{MoS}_{2}$, (d) $\mathrm{Pd}-\mathrm{MoS}_{2}$, (e) $\mathrm{Ti}_{-} \mathrm{MoS}_{2}$, (f) $\mathrm{Au}-\mathrm{WSe}_{2}$, (g) In-WSe ${ }_{2}$, (h) Pd-WSe ${ }_{2}$, (i) Ti-WSe (j) W-WSe 2 . Panel (c) and the left-hand contours in (a) and (b) show average density along $x$ projected on the $y$ - $z$ plane. Panels (d)-(j) and the right-hand plots in (a) and (b) show average electron density value in the $x-y$ planes normal to the $z$ axis $\left(\rho_{e}\right) . \rho_{m}$ in each panel indicates the minimum $x-y$ plane average electron density at each interface (in units of bohr ${ }^{-3}$ ).

better (greater than $0.02 \mathrm{bohr}^{-3}$ ), due to stronger overlap. Ti-mTMD contacts have high $\rho_{m}$ values of 0.033 and $0.029 \mathrm{bohr}^{-3}$, implying that Ti has the possibility to achieve strong orbital overlaps with monolayer $\mathrm{MoS}_{2}$ and $\mathrm{WSe}_{2}$, leading to low contact resistance. Moreover, $\mathrm{Mo}-\mathrm{MoS}_{2}$ and $\mathrm{W}-\mathrm{WSe} e_{2}$ have even higher $\rho_{m}$ at the interfaces than the others, so that the Mo and $\mathrm{W}$ top contacts can be expected to have the highest electron injection efficiency among all of the top contact metals.

These predictions can also be confirmed by Mulliken population analysis. The maximum bond Mulliken populations calculated from all of the top-contact interfaces are listed and sorted in Fig. 16(a), compared with values inside

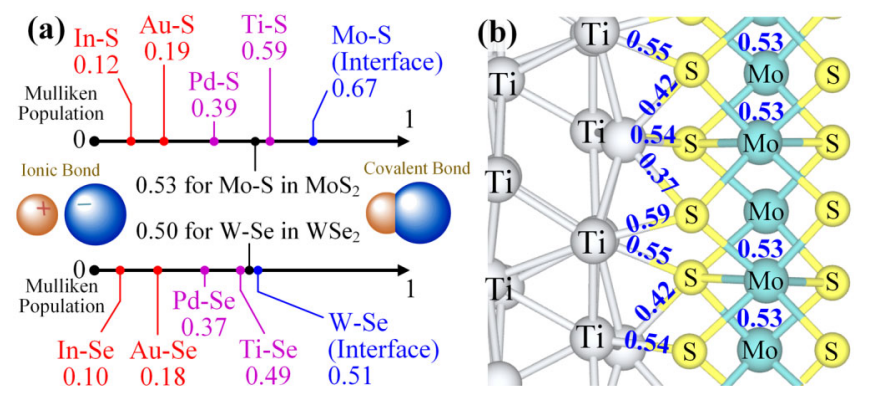

FIG. 16. (a) Maximum bond Mulliken populations (on a scale of 0 to 1 ) of top-contact interface bonds (colored numbers) compared to that of Mo-S (W-Se) bonds inside mTMDs (black numbers). (b) Bond Mulliken populations of the Ti-MoS $\mathrm{S}_{2}$ top contact. Population values are marked beside each interface bond. Population values of Mo-S bonds are also marked in $\mathrm{MoS}_{2}$. intrinsic $\mathrm{MoS}_{2}$ and $\mathrm{WSe}_{2}$. These two sequences of populations indicate the sequences of covalent bond strength. According to Fig. 16(a), the populations of Ti-S, Mo-S, and W-Se bonds at the interfaces $(0.59,0.67,0.51)$ can be even higher than that of Mo-S (W-Se) bonds inside $\mathrm{MoS}_{2}$ $\left(\mathrm{WSe}_{2}\right)$ [0.53 (0.50)], which implies that stronger covalent bonds are formed at the interfaces than in mTMDs. Particularly, to prove the strong orbital overlaps of Ti-MoS $\mathrm{S}_{2}$ top contacts, the bond Mulliken populations of all Ti-S bonds at the Ti-MoS 2 top contact interface are shown in Fig. 16(b), which range from 0.42 to 0.59 and are much higher than that for In, Au, and Pd.

\section{Summary of top contacts}

Based on the above analysis on tunnel barrier, Schottky barrier, and orbital overlap, all of the top contacts can be categorized as follows, as summarized in Fig. 17.

1. Au and In contacts are type 1. Between these two metals, Au is favored for its better orbital overlap on $\mathrm{MoS}_{2}$ [4] than that of In, while In is better in terms of Schottky barrier height and more applicable to $\mathrm{WSe}_{2}$ [7].

2. Although Pd is a type 2 metal on $\mathrm{MoS}_{2}$, the Schottky barriers from $\mathrm{Pd}$ to $\mathrm{MoS}_{2}$ are relatively higher $(0.90 \mathrm{eV}$ for both $n$ - and $p$-type SB). Hence, $\mathrm{Pd}$ is not suitable for $\mathrm{MoS}_{2}$. However, compared to the $\mathrm{Pd}-\mathrm{MoS}_{2}$ contact, $\mathrm{Pd}-\mathrm{WSe}_{2}$ provides a much lower $p$-type Schottky barrier $(0.35 \mathrm{eV})$ and much higher overlap states or metallization, which makes $\mathrm{Pd}-\mathrm{WSe}_{2}$ to be beyond type 2 and is somewhere between type 2 and type 3 .

3. Ti-MoS 2 top contact can be expected to have excellent electron injection efficiency. It can be categorized as type 3 due to the strong metallization and absence of TB at the interface. Ti also provides strong metallization to $\mathrm{WSe}_{2}$, but an unexpected tunnel barrier degrades its performance on contacting $\mathrm{WSe}_{2}$. Hence, the Ti-WSe $\mathrm{W}_{2}$ top contact is rated between type 2 and type 3 .

4. Based on DFT simulations presented above, Mo shows great potential as a high-quality contact metal for $\mathrm{MoS}_{2}$ and is classified as type 3. Mo is superior in terms of Schottky barrier $(0.13 \mathrm{eV})$ than that of Ti-MoS 2 top contact $(0.33 \mathrm{eV})$. However, the PDOS near the Fermi level of $\mathrm{MoS}_{2}$ under the Mo contact [marked by the purple brace in Fig. 11(f)] is slightly lower than that of $\mathrm{MoS}_{2}$ under the Ti contact [Fig. 11(e)], which may counter Mo's advantage of lower Schottky barrier compared to that in the case of Ti. This is in agreement with our recent measurements on Mo-MoS 2 contacts [38], where Mo and Ti contacts exhibit very similar contact resistances.

5. Similar to $\mathrm{Mo}-\mathrm{MoS}_{2}, \mathrm{~W}-\mathrm{WSe}_{2}$ is also an excellent type 3 contact. Despite its high WF, W provides $n$-type contact on $\mathrm{WSe}_{2}$ due to Fermi level pinning since it forms an alloy whose WF is close to the electron affinity of $\mathrm{WSe}_{2}$.

\section{E. Exploration of edge contacts}

Edge contacts become more important when the topcontact area is limited or in multilayered structures 
TABLE I. Evaluation of tunnel barriers at metal-mTMD edge-contact interfaces.

\begin{tabular}{lcccccccc}
\hline \hline & \multicolumn{4}{c}{$\mathrm{MoS}_{2}$} & \multicolumn{4}{c}{$\mathrm{WSe}_{2}$} \\
& $\mathrm{In}$ & $\mathrm{Ti}$ & $\mathrm{Au}$ & $\mathrm{Pd}$ & $\mathrm{In}$ & $\mathrm{Ti}$ & $\mathrm{Au}$ & $\mathrm{Pd}$ \\
\hline$d(\AA)$ & 1.38 & 0.84 & 1.70 & 1.72 & 1.54 & 1.32 & 2.18 & 1.89 \\
$\Phi_{\mathrm{TB}, \text { eff }}(\mathrm{Ry})$ & 0.00 & 0.00 & 0.54 & 0.12 & 0.00 & 0.00 & 0.12 & 0.00 \\
\hline \hline
\end{tabular}

$[12,39,40]$. In real situations, edge contacts can be induced by proper layout design. Moreover, edge contacts can be more significant for metal contacts to multilayer TMDs than that to mTMDs because more edges can be contacted to metals.

In this work, $\mathrm{Au}, \mathrm{In}, \mathrm{Pd}$, and $\mathrm{Ti}$ are chosen for modeling edge-contact configurations. In all of the simulated metal-mTMD edge contacts, the physical separations $(d)$ are much smaller (Table I) than that of top contacts [Fig. 10(b)], because of the formation of covalent bonds between the metal and mTMD as shown in Fig. 18, where overlaps of metal and chalcogenide atomic spheres can be found in each of the edge contacts. Hence, tunnel barrier widths are reduced using edge-contact configuration and edge contacts have a high possibility of covalent bond formation between metals and mTMDs.

As shown in Fig. 19, $V_{\text {eff }}$ 's along the $z$ axis are smoother at the interfaces with smaller tunnel barriers (Table I) compared to those for top contacts. For example, the $\mathrm{Au}-\mathrm{MoS}_{2}$ top contact has an effective tunnel barrier height of 0.67 Ry [Fig. 10(a)], while the height in the Au-MoS edge contact is reduced to 0.54 Ry [Fig. 19(a)], which allows higher electron injection efficiency than that of top contacts. Moreover, in the In-MoS $\mathrm{S}_{2}$ edge contact, the $\Phi_{\mathrm{TB}, \text { eff }}$ vanishes [Fig. 19(b)], compared to that of the top contact. This phenomenon is also found in Pd edge contacts.

PDOS of edge contacts are shown in Fig. 20. SBs and band gaps are absent in all of the edge contacts. The metallization is mainly due to stronger orbital overlaps, which induce high density of states in the original band gaps.

\begin{tabular}{|c|c|c|c|c|c|}
\hline \multirow[b]{2}{*}{ Criteria } & \multicolumn{2}{|c|}{ Low-WF Metals } & \multirow{2}{*}{ 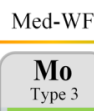 } & \multicolumn{2}{|c|}{ High-WF Metals } \\
\hline & $\underset{\text { Type } 1}{\text { In }}$ & $\underset{\text { Type } 3}{\mathbf{T i}}$ & & $\begin{array}{c}\text { Au } \\
\text { Type 1 }\end{array}$ & $\underset{\text { Type } 2}{\mathbf{P d}}$ \\
\hline Tunnel barrier & High & Small & Small & Large & Medium \\
\hline Schottky barrier & Medium & $\operatorname{Low}(n)$ & Low(n) & Medium & High \\
\hline Orbital overlap & Weak & Strong & Strong & Weak & Medium \\
\hline Criteria & $\underset{\text { Type } 1}{\operatorname{In}}$ & $\underset{\text { Type 2-3 }}{\mathrm{Ti}}$ & $\underset{\text { Type } 3}{\mathbf{W}}$ & $\underset{\text { Type } 1}{\text { Au }}$ & $\underset{\text { Type 2-3 }}{\mathbf{P d}}$ \\
\hline Tunnel barrier & High & Medium & Small & Large & Medium \\
\hline Schottky barrier & Low(n) & Low(n) & Low(n) & Medium & Low(p) \\
\hline Orbital overlap & Weak & Strong & Strong & Weak & Strong \\
\hline
\end{tabular}

FIG. 17. Summary of metal-mTMD top-contact electron injection efficiency, in terms of orbital overlap, Schottky barrier, and tunnel barrier.
Minimum valence electron densities at the interfaces $\left(\rho_{\min }\right)$ are increased compared to that of most of the top contacts, as shown in Figs. 21(a) and 21(b), due to the strong orbit overlap (covalent bonds) between metal and mTMD atoms. In particular, $\rho_{\min }$ in $\mathrm{Au}-\mathrm{MoS}_{2}$ [Figs. 21(c) and 21(d)] and In-mTMD edge contacts are significantly increased compared to that of their top contacts [Figs. 15(a), 15(b), and 15(g)], indicating a decrease in resistance by changing contact configurations from top contacts into combined contacts [Fig. 3(c)]. Low contact resistance of combined-contact configuration has been demonstrated on Au-MoS 2 [4], Ti-MoS 2 [12] and In-WSe $\mathrm{W}_{2}$ [7] contacts via experiments.

Furthermore, the electron localization functions (ELF, a function of the 3D coordinates, which is large in the regions where orbitals localize [41]) for $\mathrm{Au}-\mathrm{MoS}_{2}$ top and edge contacts [Figs. 22(a) and 22(c)] are calculated and shown in Figs. 22(b) and 22(d). Because orbital overlaps between metal and mTMD atoms are more efficient in edge contacts, the ELF at the metal-mTMD interface is much higher at the

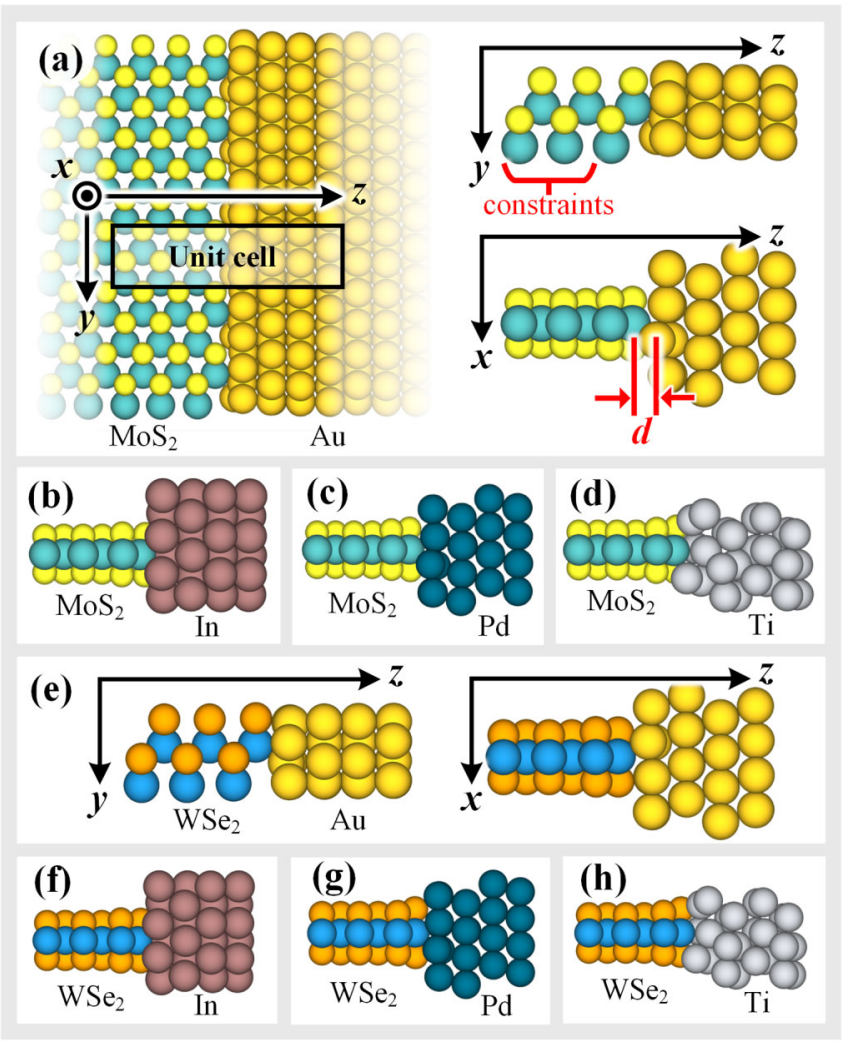

FIG. 18. Optimized geometries of edge contacts: (a) Au-MoS (in different views), (b) In- $\mathrm{MoS}_{2}$, (c) $\mathrm{Pd}-\mathrm{MoS}_{2}$, (d) $\mathrm{Ti}-\mathrm{MoS}_{2}$, (e) $\mathrm{Au}-\mathrm{WSe}_{2}$ (in different views), (f) In-WSe 2 , (g) Pd-WSe ${ }_{2}$, and (h) $\mathrm{Ti}_{-} \mathrm{WSe}_{2}$. The large-volume overlaps of metal and chalcogenide atomic spheres exist in every edge contact [in (a)-(h)], indicating the formation of strong covalent bonds. Hence, physical separations in edge contacts (Table I) are much smaller than that of top contacts in Figs. 8 and 9 due to the covalent bonds formed between mTMD and metals. 


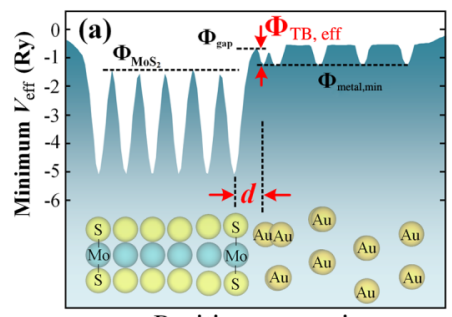

Position on $z$ axis

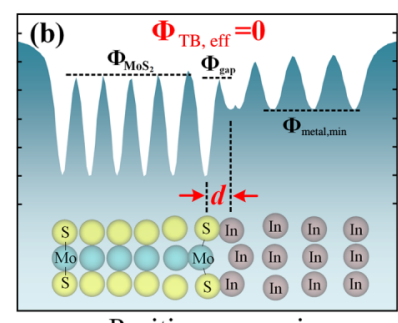

Position on $z$ axis

FIG. 19. Minimum $V_{\text {eff }}$ of (a) Au-MoS 2 edge contact and (b) In-MoS 2 edge contact. $\Phi_{\mathrm{MoS}_{2}}$ is the $V_{\text {eff }}$ of the Mo-S bond orbitals and thereby the effective tunnel barrier height $\left(\Phi_{\mathrm{TB} \text {,eff }}\right)$ is defined as the minimum barrier height that an electron from the metal has to overcome, if it has the same potential energy as $\Phi_{\mathrm{MoS}_{2}}$. Hence, $\Phi_{\mathrm{TB}, \text { eff }}$ can be calculated as the $V_{\text {eff }}$ difference between the $\operatorname{vdW}$ gap $\left(\Phi_{\text {gap }}\right)$ and $\operatorname{MoS}_{2}\left(\Phi_{\mathrm{MoS}_{2}}\right) . \Phi_{\text {metal,min }}$ denotes the minimum $V_{\text {eff }}$ that an electron can have in the metal. It is worth noting that in some metals [such as in (a)] $\Phi_{\text {metal,min }}$ can be higher than $\Phi_{\mathrm{MoS}_{2}}$ (thus, electron energy is always higher than that of Mo-S bond orbitals), in which case $\Phi_{\mathrm{TB}, \text { eff }}$ is calculated as $\Phi_{\mathrm{TB}, \text { eff }}=\Phi_{\text {gap }}-\Phi_{\text {metal,min }} . d$ is defined as the physical separation (the $z$ component of the nearest core-to-core distance between the metal atoms and the chalcogenide atoms). Though $d$ does not vanish, $\Phi_{\mathrm{TB}, \text { eff }}$ can vanish, when $\Phi_{\text {metal,min }}$ or $\Phi_{\mathrm{MoS}_{2}}$ is higher than $\Phi_{\text {gap }}$, such as in (b).

interface in edge contacts [Fig. 22(d)] than that of top contacts [Fig. 22(b)]. In other words, strong covalent bonds are formed in edge contacts, indicated by green dashed curves in Fig. 22(d).

The strong orbital overlaps, absence of SB, and lower tunnel barriers are all advantages of edge-contact configurations compared to top contacts. Hence, edge-contacted configurations have a higher capability of electron injection and thereby decrease the contact resistance. If contact dimensions are large (i.e., number of atoms across the

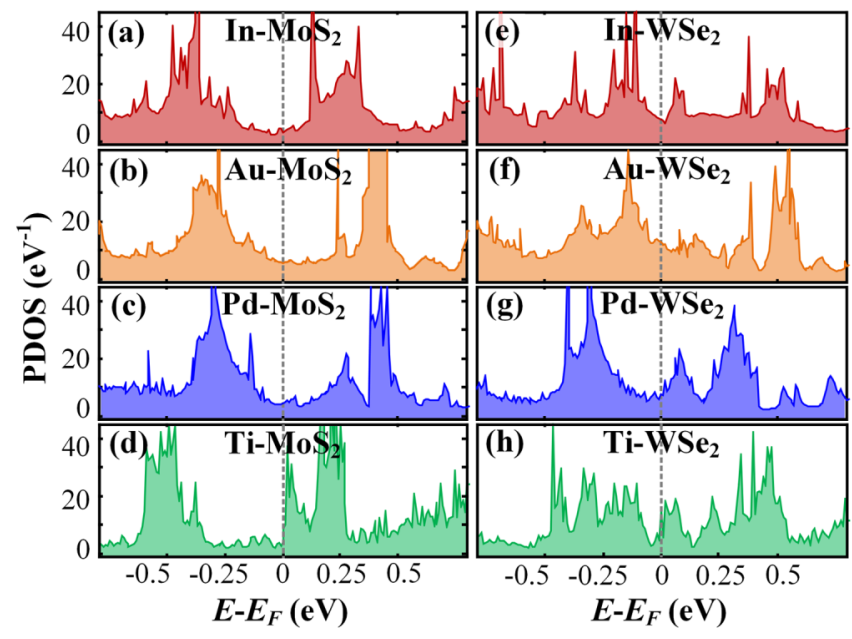

FIG. 20. PDOS of mTMD near $E_{F}$ of edge contacts to (a)(d) $\mathrm{MoS}_{2}$ and (e)-(h) $\mathrm{WSe}_{2}$. Because of orbital overlaps (covalent bonds) at the interfaces, all the mTMDs in edge contacts have overlap states in the original band gaps and near $E_{F}$, so that mTMDs are metallized by edge contacts.
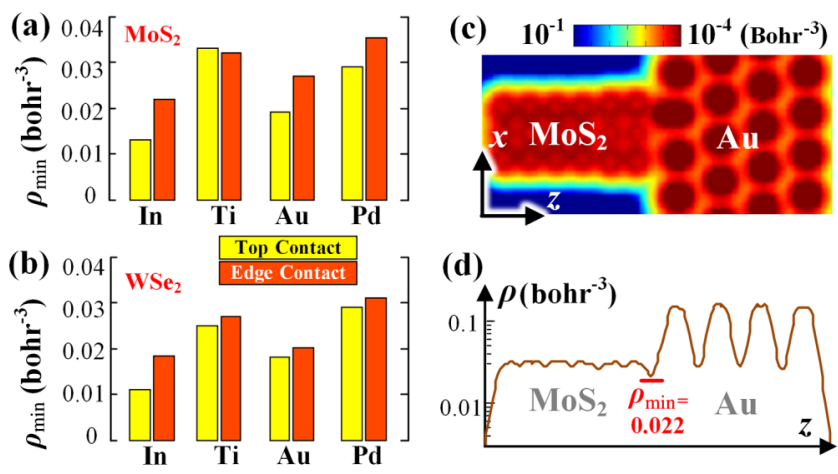

FIG. 21. (a),(b) Minimum of average electron density values in the $x$ - $y$ plane in Fig. 18(a) at the interfaces for all top and edge contacts. Ti, Pd, Mo, and $\mathrm{W}$ may form better top contacts due to higher interface electron density. Electron densities are significantly increased for edge contacts. (c),(d) Valence electron densities of $\mathrm{Au}-\mathrm{MoS}_{2}$ edge contact. (c) Average density along $y$ projected on the $x-z$ plane; (d) average density in the $x-y$ planes normal to the $z$ axis. The red number in (d) indicates the minimum electron density at the interface.

contact area is much greater than those along the contact perimeter), top contacts have an advantage in terms of contact area. However, for a fixed number of mTMD atoms contacted by metal, the edge-contacted configuration is better than the top-contacted configuration. Hence, the combined contact [Fig. 3(c), combination of top contact and edge contact] is more desirable. It is worth noting that for multilayer TMD devices, due to process voids, the electrode metal may fail to contact all of the layers at the edges, and the gate electrode may not modulate all the layers due to screening. Therefore, the number of TMD layers should be optimized [12].
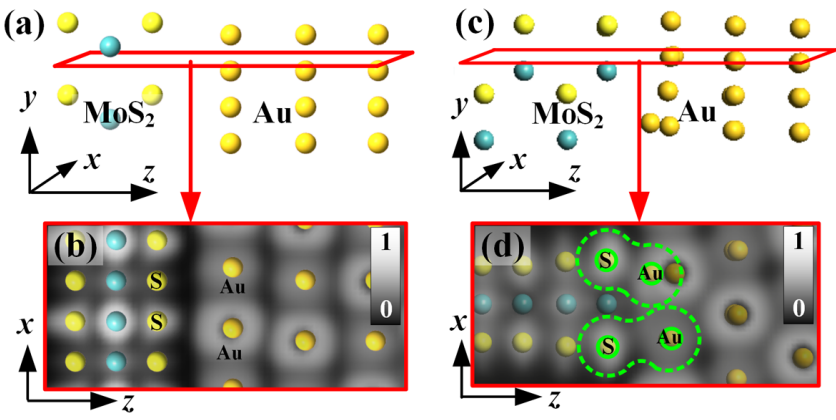

FIG. 22. (a),(c) The cleaved $x-z$ planes (red rectangles) used to show electron localization functions (ELF) for (a) Au-MoS $\mathrm{M}_{2}$ top contact and (c) Au-MoS 2 edge contact. (b),(d) ELF plots on the cleaved $x-z$ plane in $\mathrm{Au}-\mathrm{MoS}_{2}$ (b) top contact, (d) edge contact. Half-transparent dots indicate positions of atoms. High ELF (closer to 1) indicates high probability of finding an electron. For edge contact, $\mathrm{Au}-\mathrm{MoS}_{2}$ has overlapping electron orbitals (Au-S bonds, indicated by green dashes) resulting in the increasing of electron density as shown in Fig. 21. Thus, the contact resistance is reduced by using edge contacts. 


\section{SUMMARY}

This work presented a systematic and rigorous study of the physical nature of metal-mTMD interfaces. The electron injection efficiency of the interfaces is shown to be characterized by three key criteria-tunnel barrier, Schottky barrier, and orbital overlap. In order to accurately capture each of those criteria, DFT simulations incorporating semiempirical vdW potential are employed for the first time for metal-mTMD interfaces, and optimized geometries, effective potential, band structures, PDOS, valence electron densities, and bond Mulliken populations of metal-mTMD contacts are calculated.

We find that $\mathrm{Ti}$ and Mo are the best top-contact metals for monolayer intrinsic $\mathrm{MoS}_{2}$ and are $n$-type contact metals (Fig. 17). Pd is the best $p$-type top-contact metal for monolayer intrinsic $\mathrm{WSe}_{2}$ (Fig. 17) while $\mathrm{W}$ can achieve high-quality $n$-type top contacts with $\mathrm{WSe}_{2}$ due to the strong orbital overlaps and vanishing of Schottky barriers (Fig. 17). These findings are summarized and shown in Fig. 17.

While none of the metals studied in this work indicate the capability of forming good $p$-type contacts to $\mathrm{MoS}_{2}$, from the basic interface physics revealed in this study, materials with strong orbital overlaps with $\mathrm{MoS}_{2}$ have the potential to lead to such contacts. Such properties can possibly be found in molybdenum oxide compounds $\left(\mathrm{MoO}_{x}\right)$.

It is also shown that edge-contacted configurations can improve the contact by lowering tunnel barriers and strengthening the orbital overlaps. With the right metal and certain contact area, in order to achieve the lowest contact resistance, it is desirable to combine edge contact with top contact for monolayer TMDs [Fig. 3(c)]. It can be inferred that inducing of edge contacts can be more significant for multilayer mTMDs. For more-than-ten-layer TMDs, it is necessary to ensure that all of the edges are contacted to the metal using the tilt deposition technique [42]. On the other hand, it is possible to increase the edgecontact length for lower contact resistance, for example, by cutting mTMD edges into jagged edges.

The results obtained in this study not only reveal the types of metals and configurations that can be employed for achieving low contact resistance with $\mathrm{MoS}_{2}$ and $\mathrm{WSe}_{2}$, but also highlight that the properties of contacts cannot be intuitively predicted by solely considering WF values (e.g., $\mathrm{Au}$ versus Pd; In versus Ti; Mo or W versus other metals). Moreover, the significance of the developed framework, which features vdW interactions and bond Mulliken population analysis is apparent not only for contacts to various 2D materials, but also for understanding the nature of interfaces to a wide variety of 2D materials, which will be a key issue in optimizing the performance of all emerging $2 \mathrm{D}$ materials-based devices including the recently proposed concept of "all-2D devices and circuits" [43] (where graphene is used as gate electrodes and interconnects, $\mathrm{MoS}_{2}$ and $\mathrm{WSe}_{2}$ are used as channel materials in the FETs, and insulating $h$-BN is used as a gate dielectric). By

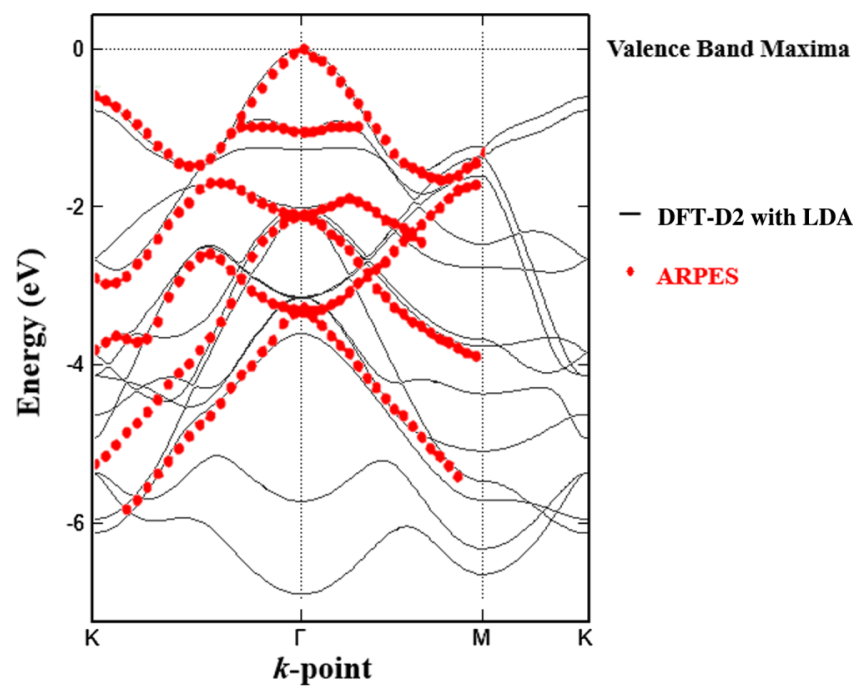

FIG. 23. Comparison of calculated (DFT-D2 with LDA) and experimentally measured (ARPES) [44] valence band structures of $\mathrm{MoS}_{2}$. The valence band maxima is set to zero energy.

combining our framework and transport simulations, quantitative values of contact resistances can be calculated in the future.

\section{ACKNOWLEDGMENTS}

This work was supported by the National Science Foundation (Grant No. CCF-1162633).

\section{APPENDIX: BAND STRUCTURE EVALUATION FOR DFT-D2 WITH LDA}

As pointed out in Section II C, the DFT-D2 results show consistent bandgaps with results from experiments $[25,26]$. Since the valence band structures of bulk $\mathrm{MoS}_{2}$ were recently measured by angle-resolved photoemission spectroscopy (ARPES) as reported in [44], to further evaluate the simulation methodology, the calculated valence band structures of bulk $\mathrm{MoS}_{2}$ by DFT-D2 with LDA (black curves) are superimposed on that of ARPES results (red dots) from [44], as shown in Fig. 23. It can be observed that the shapes of electronic dispersion spectrum of $\mathrm{MoS}_{2}$ in simulations are in agreement with those from the experiments.

This provides strong evidence that the methodology employed in this work can reproduce the electronic spectrum correctly and the agreements between simulations and experiments are not just coincidental.

[1] K. S. Novoselov, A. K. Geim, S. V. Morozov, D. Jiang, Y. Zhang, S. V. Dubonos, I. V. Grigorieva, and A. A. Firsov, Electric Field Effect in Atomically Thin Carbon Films, Science 306, 666 (2004).

[2] A. K. Geim and K. S. Novoselov, The Rise of Graphene, Nat. Mater. 6, 183 (2007). 
[3] G.-H. Lee, Y.-J. Yu, C. Lee, C. Dean, K. L. Shepard, P. Kim, and J. Hone, Electron Tunneling through Atomically Flat and Ultrathin Hexagonal Boron Nitride, Appl. Phys. Lett. 99, 243114 (2011).

[4] B. Radisavljevic, A. Radenovic, J. Brivio, V. Giacometti, and A. Kis, Single-Layer $\mathrm{MoS}_{2}$ Transistors, Nat. Nanotechnol. 6, 147 (2011).

[5] Y. Yoon, K. Ganapathi, and S. Salahuddin, How Good Can Monolayer $\mathrm{MoS}_{2}$ Transistors Be?, Nano Lett. 11, 3768 (2011).

[6] H. Fang, S. Chuang, T.C. Chang, and K. Takei, High-Performance Single Layered $\mathrm{WSe}_{2}$ P-FETs with Chemically Doped Contacts, Nano Lett. 12, 3788 (2012).

[7] W. Liu, J. Kang, D. Sarkar, Y. Khatami, D. Jena, and K. Banerjee, Role of Metal Contacts in Designing HighPerformance Monolayer n-Type $\mathrm{WSe}_{2}$ Field Effect Transistors, Nano Lett. 13, 1983 (2013).

[8] S. Kim, A. Konar, W. Hwang, J. H. Lee, J. Lee, J. Yang, C. Jung, H. Kim, J. Yoo, J. Choi, Y. W. Jin, S. Y. Lee, D. Jena, W. Choi, and K. Kim, High-Mobility and Low-Power ThinFilm Transistors based on Multilayer $\mathrm{MoS}_{2}$ Crystals, Nat. Commun. 3, 1011 (2012).

[9] D. Sarkar, W. Liu, X. Xie, A. Anselmo, S. Mitragotri, and K. Banerjee, $\mathrm{MoS}_{2}$ Field-Effect Transistor for NextGeneration Label-Free Biosensors, ACS Nano 8, 3992 (2014).

[10] I. Popov, G. Seifert, and D. Tománek, Designing Electrical Contacts to $\mathrm{MoS}_{2}$ Monolayers: A Computational Study, Phys. Rev. Lett. 108, 156802 (2012).

[11] S. Das, H.-Y. Chen, A. V. Penumatcha, and J. Appenzeller, High Performance Multilayer $\mathrm{MoS}_{2}$ Transistors with Scandium Contacts, Nano Lett. 13, 100 (2013).

[12] W. Liu, J. Kang, W. Cao, D. Sarkar, Y. Khatami, D. Jena, and K. Banerjee, in Proceedings of the IEEE International Electron Devices Meeting, 2013 (IEEE, New York, 2013), pp. 499-502.

[13] L.-Y. Gan, Y.-J. Zhao, D. Huang, and U. Schwingenschlögl, First-Principles Analysis of $\mathrm{MoS}_{2} / \mathrm{Ti}_{2} \mathrm{C}$ and $\mathrm{MoS}_{2} / \mathrm{Ti}_{2} \mathrm{CY}_{2}$ $(Y=\mathrm{F}$ and $\mathrm{OH}$ ) All-2D Semiconductor/Metal Contacts, Phys. Rev. B 87, 245307 (2013).

[14] International Technology Roadmap for Semiconductors, http://www.itrs.net/.

[15] H. Liu, A. T. Neal, and P. D. Ye, Channel Length Scaling of $\mathrm{MoS}_{2}$ MOSFETs, ACS Nano 6, 8563 (2012).

[16] E. K. U. Gross and R. M. Dreizler, Density Functional Theory (Plenum, New York, 1993).

[17] J. Kang, D. Sarkar, W. Liu, D. Jena, and K. Banerjee, in Proceedings of the IEEE International Electron Devices Meeting, 2012 (IEEE, New York, 2012), pp. 407-410.

[18] Handbook of Chemistry and Physics, edited by V. R. C. Weast (CRC Press, Boca Raton, FL, 1979), 59th ed., p. 114.

[19] P. Bhattacharya, Semiconductor Optoelectronic Devices (Prentice-Hall, Englewood Cliffs, NJ, 1997), 2nd ed.

[20] Y. Li, Z. Zhou, S. Zhang, and Z. Chen, $\mathrm{MoS}_{2}$ Nanoribbons: High Stability and Unusual Electronic and Magnetic Properties, J. Am. Chem. Soc. 130, 16739 (2008).

[21] Y. Matsuda, W.-Q. Deng, and W. A. Goddard, Contact Resistance for "End-Contacted" Metal-Graphene and Metal-Nanotube Interfaces from Quantum Mechanics, J. Phys. Chem. C 114, 17845 (2010).
[22] W. Kohn and L. J. Sham, Self-Consistent Equations Including Exchange and Correlation Effects, Phys. Rev. 140, A1133 (1965).

[23] J. P. Perdew, K. Burke, and M. Ernzerhof, Generalized Gradient Approximation Made Simple, Phys. Rev. Lett. 77, 3865 (1996); 78, 1396(E) (1997).

[24] J. P. Perdew and A. Zunger, Self-Interaction Correction to Density-Functional Approximations for Many-Electron Systems, Phys. Rev. B 23, 5048 (1981).

[25] K. F. Mak, C. Lee, J. Hone, J. Shan, and T. F. Heinz, Atomically Thin $\mathrm{MoS}_{2}$ : A New Direct-Gap Semiconductor, Phys. Rev. Lett. 105, 136805 (2010).

[26] D. Vo $\beta$, P. Krüger, A. Mazur, and J. Pollmann, Atomic and Electronic Structure of $\mathrm{WSe}_{2}$ from Ab Initio Theory: Bulk Crystal and Thin Film Systems, Phys. Rev. B 60, 14311 1999).

[27] M. Vanin, J. J. Mortensen, A. K. Kelkkanen, J. M. GarciaLastra, K. S. Thygesen, and K. W. Jacobsen, Graphene on Metals: A van der Waals Density Functional Study, Phys. Rev. B 81, 081408 (2010).

[28] T. Björkman, A. Gulans, A. V. Krasheninnikov, and R. M. Nieminen, Are We van der Waals Ready?, J. Phys. Condens. Matter 24, 424218 (2012).

[29] T. Björkman, A. Gulans, A. V. Krasheninnikov, and R. M. Nieminen, van der Waals Bonding in Layered Compounds from Advanced Density-Functional First-Principles Calculations, Phys. Rev. Lett. 108, 235502 (2012).

[30] S. Grimme, Accurate Description of van der Waals Complexes by Density Functional Theory Including Empirical Corrections, J. Comput. Chem. 25, 1463 (2004).

[31] S. Grimme, Semiempirical GGA-Type Density Functional Constructed with a Long-Range Dispersion Correction, J. Comput. Chem. 27, 1787 (2006).

[32] S. Grimme, J. Antony, S. Ehrlich, and H. Krieg, A Consistent and Accurate Ab Initio Parametrization of Density Functional Dispersion Correction (DFT-D) for the 94 Elements $\mathrm{H}-\mathrm{Pu}$, J. Chem. Phys. 132, 154104 (2010).

[33] Y. Andersson, D. C. Langreth, and B. I. Lundqvist, van der Waals Interactions in Density-Functional Theory, Phys. Rev. Lett. 76, 102 (1996).

[34] D. C. Langreth, M. Dion, H. Rydberg, E. Schröder, P. Hyldgaard, and B. I. Lundqvist, van der Waals Density Functional Theory with Applications, Int. J. Quantum Chem. 101, 599 (2005).

[35] T. Sato, T. Tsuneda, and K. Hirao, van der Waals Interactions Studied by Density Functional Theory, Mol. Phys. 103, 1151 (2005).

[36] Atomistix ToolKit v.13.8.0, QuantumWise A/S, http://www .quantumwise.com/

[37] R. S. Mulliken, Electronic Population Analysis on LCAOMO Molecular Wave Functions. I, J. Chem. Phys. 23, 1833 (1955).

[38] J. Kang, W. Liu, and K. Banerjee, High-Performance $\mathrm{MoS}_{2}$ Transistors with Low-Resistance Molybdenum Contacts, Appl. Phys. Lett. 104, 093106 (2014).

[39] Y. Khatami, H. Li, C. Xu, and K. Banerjee, Metal-toMultilayer-Graphene Contact-Part I: Contact Resistance Modeling, IEEE Trans. Electron Devices 59, 2444 (2012).

[40] Y. Khatami, H. Li, C. Xu, and K. Banerjee, Metal-toMultilayer-Graphene Contact-Part II: Analysis of Contact 
Resistance, IEEE Trans. Electron Devices 59, 2453 (2012).

[41] A. D. Becke and K. E. Edgecombe, A Simple Measure of Electron Localization in Atomic and Molecular Systems, J. Chem. Phys. 92, 5397 (1990).

[42] H. Li, W. Liu, A. M. Cassell, F. Kreupl, and K. Banerjee, Low-Resistivity Long-Length Horizontal Carbon Nanotube Bundles for Interconnect Applications-Part I: Process Development, IEEE Trans. Electron Devices 60, 2862 (2013).
[43] W. Cao, J. Kang, W. Liu, Y. Khatami, D. Sarkar, and K. Banerjee, in Proceedings of the 43rd European Solid-State Device Research Conference (ESSDERC), Bucharest, Romania, 2013 (IEEE, New York, 2013), pp. 37-44.

[44] S. K. Mahatha, K. D. Patel, and K. S. R. Menon, Electronic Structure Investigation of $\mathrm{MoS}_{2}$ and $\mathrm{MoSe}_{2}$ Using AngleResolved Photoemission Spectroscopy and Ab Initio Band Structure Studies, J. Phys. Condens. Matter 24, 475504 (2012). 